Artículo

\title{
Transición demográfica y sus consecuencias en la matrícula universitaria en Colombia*
}

\section{Demographic Transition and its Consequences on University Enrollment in Colombia}

\author{
Adolfo Meisel Roca $[$ y Angela Granger $(\mathbb{0}$ \\ ameisel@uninorte.edu.co; agranger@uninorte.edu.co \\ Universidad del Norte, Barranquilla, Colombia
}

\begin{abstract}
Resumen
La transición demográfica en Colombia, así como en el mundo, ha reestructurado la composición por edad de la población y ha impuesto nuevos retos a diversos sectores de la sociedad. En este trabajo analizamos este fenómeno y sus efectos sobre la demanda por educación superior y, en particular, sobre la población universitaria. A través del análisis de datos censales, registros de las pruebas Saber 11 y datos del Sistema Nacional de Información de la Educación Superior (SNIES), se observa una disminución tanto del tamaño de las cohortes de estudiantes que ingresan y finalizan el bachillerato como de la demanda por educación superior. El argumento que explica estos hechos, que además difieren entre regiones, gira alrededor de la disminución de la fecundidad en Colombia.
\end{abstract}

Palabras clave: Transición demográfica, fecundidad, educación superior, demanda, universidades, regiones.

Clasificaciones JEL: I20, I21, J11, J18.

\footnotetext{
* Cómo citar este artículo: Meisel Roca, A., y Granger, A. (2020). Transición demográfica y sus consecuencias en la matrícula universitaria en Colombia. Economía E Región, 14(1), 1-34. https:/ / doi.org/10.32397/er.vol14.n1.1

Recibido: 15-jul-2020; aceptado: 29-ene-2021; publicado: 27-jul-2021

revistas.utb.edu.co/index.php/economiayregion
} 


\begin{abstract}
Demographic transition in Colombia, as well as in the world, has restructured the age composition of the population and has imposed new challenges on various sectors of society. In this paper we analyze this phenomenon and its effects on the demand for higher education and, in particular, on the university population. Through the analysis of census data, records of the Saber 11 tests and data from the National Information System for Higher Education (SNIES in spanish), we find a decrease in both the size of the cohorts of students entering and completing high school as well as the demand for higher education. The argument that explains these facts, which also differ between regions, revolves around the decline in fertility in Colombia.
\end{abstract}

Keywords: demographic transition, fertility, higher education, demand, universities, regions.

JEL classifications: I20, I21, J11, J18.

\title{
I. INTRODUCCIÓN
}

Hasta comienzos del siglo XIX a nivel mundial el crecimiento económico y demográfico de largo plazo fue cercano a cero. La historia demográfica y económica hasta la Revolución Industrial se describe como la trampa maltusiana: sociedades en las que los aumentos en el producto per cápita desaparecían rápidamente con el crecimiento poblacional. Sin embargo, las trasformaciones que sobrevinieron con la Revolución Industrial permitieron la salida de esa trampa maltusiana. La creciente urbanización, las reformas a los sistemas políticos, las mejores condiciones sanitarias y los avances en la producción a gran escala de alimentos permitieron la reducción de las tasas de mortalidad y dieron paso al crecimiento económico y poblacional (Clark, 2007). Entre los siglos XIX y XX, la población europea se multiplicó por cuatro, resultado de la profunda transformación social que se inició a finales del siglo XVIII. Por eso, desde el siglo XIX, se observó un enorme crecimiento de la población mundial (Livi-Bacci, 2017).

Ya en el siglo XX, la explosión demográfica causó preocupación acerca de la sostenibilidad del crecimiento económico. Sin embargo, no pasaría mucho tiempo antes de que los países vivieran un nuevo cambio en la tendencia del crecimiento poblacional, esta vez dada por el descenso en la tasa de natalidad por causa de una mayor supervivencia de los niños nacidos, la creciente participación laboral de la mujer y la mayor disponibilidad de métodos anticonceptivos. Este proceso mediante el cual las sociedades pasan de altas a bajas tasas de mortalidad y luego disminuye también su tasa de natalidad se conoce como la transición demográfica, uno de los hitos centrales de la historia económica mundial en los últimos 250 años. 
Así como en América Latina y en el mundo, en Colombia también se perciben las consecuencias económicas y sociales de un proceso avanzado de transición demográfica. Ello ocurre en temas como las pensiones, el crecimiento de la población y la demanda por la educación superior. El cambio en la estructura por edades de la población, aunque no es el único determinante, tiene un rol principal en la demanda por educación. En este trabajo, analizamos este fenómeno demográfico y sus consecuencias sobre la población universitaria. Para ello, en la siguiente sección examinamos las principales transformaciones demográficas de los países latinoamericanos y su contraste con los países desarrollados. Seguido, se analiza el caso colombiano con una visión general y regional de la transición demográfica. En las siguientes dos secciones se presentan los efectos sobre la demanda por educación universitaria dada la evidencia demográfica reciente. Y, finalmente, concluimos con los retos que enfrentan las instituciones educativas y con sugerencias de política pública frente a un país con cada vez menos jóvenes.

\section{TRANSICIÓN DEMOGRÁFICA EN AMÉRICA LATINA Y EN LOS PAÍSES MIEMBROS DE LA ORGANIZACIÓN PARA LA COOPERACIÓN Y EL DESARROLLO ECONÓMICO (OCDE)}

En la actualidad, los países desarrollados se encuentran en una etapa avanzada de la transición demográfica y enfrentan los desafíos que supone el envejecimiento de la población. A su vez, los países en desarrollo, y en particular los de América Latina y el Caribe, aunque iniciaron rezagados el proceso de transición demográfica, han avanzado aceleradamente en el incremento de la esperanza de vida y en la disminución de la fecundidad. Lo que a los primeros países les tomó siglos (y consiguieron a través del crecimiento económico y el cambio en las condiciones de vida) a los países latinoamericanos y del Caribe insular les tomó apenas algunas décadas (aun en medio de crisis económicas y, algunos de ellos, en medio de la pobreza). Probablemente esto ocurrió, entre otras cosas, porque en el siglo XX los países en desarrollo pudieron adoptar la tecnología ya disponible para el control de la mortalidad y la natalidad (Chackiel, 2004).

Entre 1960 y 2017, la esperanza de vida promedio de los habitantes en los países miembros de la OCDE pasó de 67,4 a 80,1 años. En América Latina y el Caribe, la esperanza de vida pasó de 56 a 75,3 años durante el mismo período (Gráfico 1). Este último incremento es evidencia del enorme cambio social que ocurrió en el siglo XX y que aún continúa. A pesar de que existe gran heterogeneidad entre los países de América Latina y el Caribe, no hay duda de que todos se han incorporado al proceso de transición demográfica avanzando a la velocidad que les ha permitido su progreso social y económico.

La primera etapa de esta transición demográfica constituye un mundo con altas tasas de mortalidad y de natalidad, y por lo tanto una población mayormente estable. La primera transformación que da paso al crecimiento poblacional es la caída de la mortalidad que dio lugar a un aumento de la expectativa de vida, sobre todo debido a la caída de la mortalidad infantil desde finales del siglo XIX en los países desarrollados 
Gráfico 1. América Latina y el Caribe y países miembros de la OCDE:

Esperanza de vida al nacer, 1960 - 2017

(número de años)

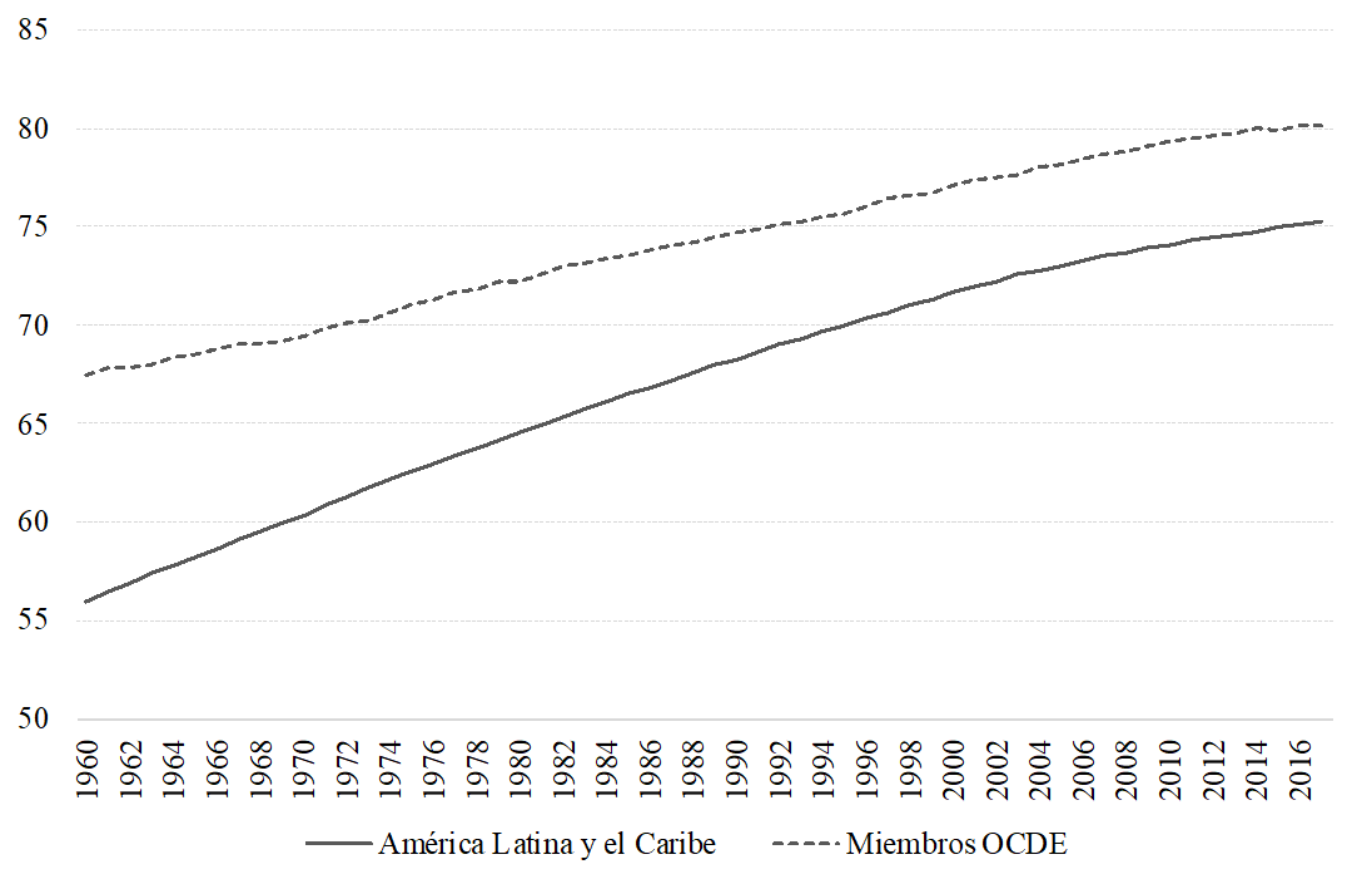

Fuente: Elaboración propia con base en datos del Banco Mundial.

y desde el siglo XX en los países en vías de desarrollo. No obstante, con el tiempo, este indicador deja de ser adecuado para analizar la transición demográfica en la medida en que el comportamiento reciente transforma la estructura de edades de la población en una con mayor longevidad y, por tanto, con mayor mortalidad en la adultez. Este fenómeno explica por qué en la década de 1970 la tasa de mortalidad en los países miembros de la OCDE sobrepasó la de América Latina y el Caribe (Gráfico 2).

La siguiente etapa de la transición demográfica se inicia con el descenso de la fecundidad. A principios de 1960 las mujeres en América Latina y el Caribe tenían en promedio 5,9 hijos, cifra que en 2017 cayó a 2,04. Los países desarrollados ya habían experimentado el descenso de la natalidad un siglo atrás y para 1960 cada mujer tenía 3,2 hijos en promedio. En 2017, eran aproximadamente 1,71 hijos por mujer, cifra por debajo de la tasa de reemplazo de la población (Gráfico 3). ${ }^{1}$ Aun cuando la transición demográfica ha sido acelerada en los países en desarrollo, para 2015 el crecimiento natural de la población en América Latina y el Caribe seguía siendo mayor que aquel de los países miembros de la OCDE en aproximadamente 0,4 puntos porcentuales (pp) (Gráfico 4).

\footnotetext{
${ }^{1}$ La tasa de reemplazo es la fecundidad mínima necesaria para que el crecimiento natural de una población sea cero. Usualmente se encuentra alrededor de 2,1 pero varía de acuerdo con las tasas de mortalidad (Searchinger, et al., 2013).
} 
Gráfico 2. América Latina y el Caribe y países miembros de la OCDE:

Tasas de natalidad y mortalidad, 1960 - 2017

(nacimientos y muertes por cada 1.000 habitantes)

45

40

35

30

25

20

15

10

5

-Mortalidad - América Latina y el Caribe -Mortalidad - OCDE

..... Natalidad - América Latina y el Caribe ...--Natalidad - OCDE

Fuente: Elaboración propia con base en datos del Banco Mundial.

Gráfico 3. América Latina y el Caribe y países miembros de la OCDE:

Tasa global de fecundidad, 1960 - 2016

(número de hijos promedio por mujer)

7

6

5

1

0

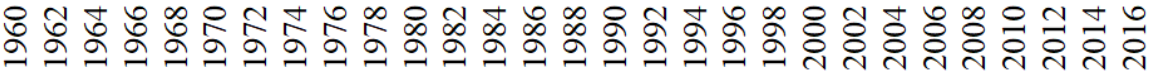

—_América Latina y el Caribe -----Miembros OCDE

Fuente: Elaboración propia con base en datos del Banco Mundial. 


\section{Gráfico 4. América Latina y el Caribe y países miembros de la OCDE: \\ Crecimiento poblacional, 1961 - 2015 \\ (porcentajes)}

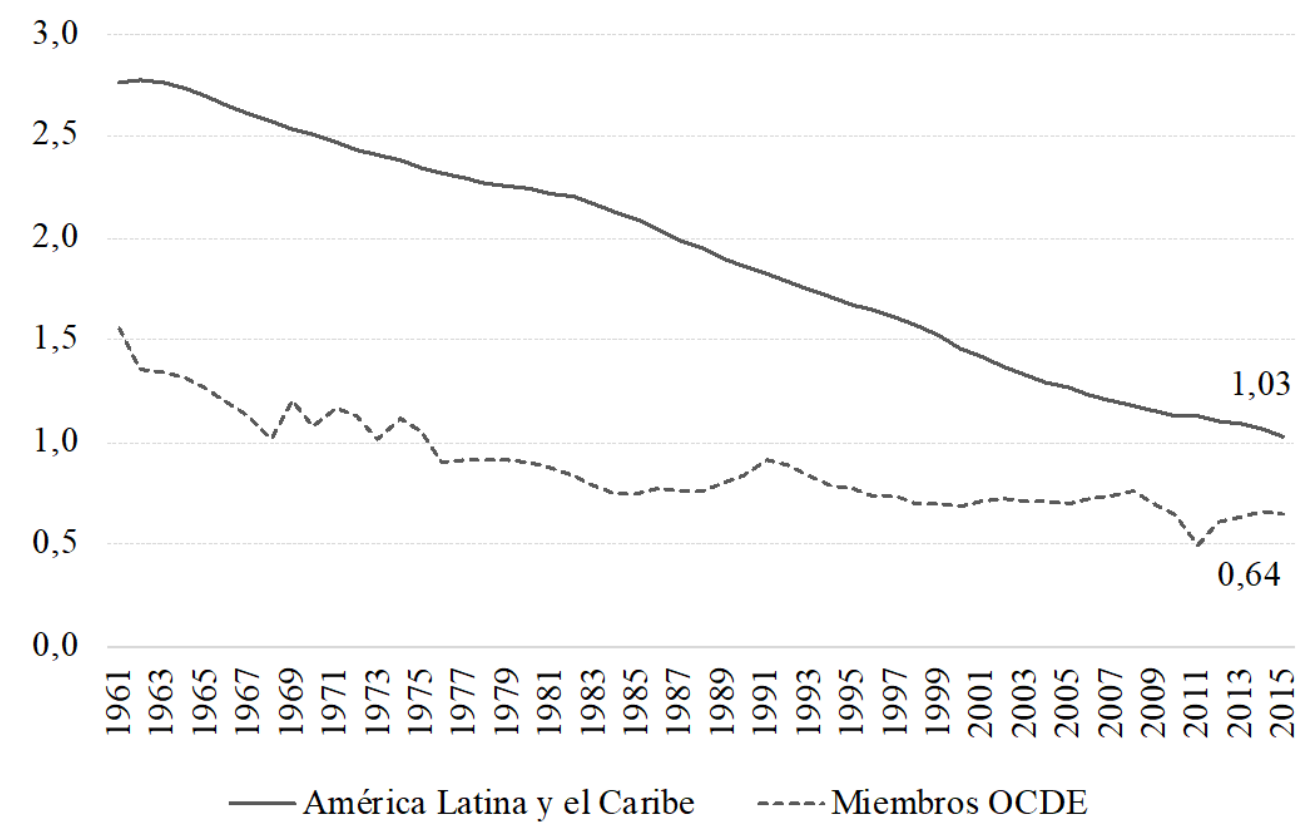

Fuente: Elaboración propia con base en datos del Banco Mundial.

El descenso de la natalidad y la mortalidad ha aproximado el crecimiento natural de la población cada vez más a cero. Incluso, en algunos países como Italia, Japón o Rusia se han observado tasas negativas. El efecto de la explosión demográfica del siglo XX, que aumentó la base de la pirámide poblacional, se ha diluido y en las últimas décadas el acelerado descenso de la fecundidad ha invertido la distribución de edades de la población con un creciente índice de envejecimiento (Gráficos 5 y 6).

A pesar de ello, este proceso de envejecimiento ha sido progresivo. La tasa de dependencia - que expresa la razón entre el número de personas dependientes (mayores de 65 años y menores de 14) por cada cien personas en edad de trabajar (entre 15 y 65 años) - es alta durante el inicio de la transición demográfica dado el creciente número de niños que sobreviven. En 1960, en América Latina y el Caribe, había 85,4 personas dependientes por cada 100 personas en edad de trabajar (75,7 jóvenes y 6,6 adultos mayores). A partir de 1967, el índice decreció continuamente hasta 49,2 en 2018, con una relación entre adultos mayores y jóvenes cercana a 3:1 (36,6 jóvenes y 12,6 adultos mayores). Al inicio del período, los países miembros de la OCDE tuvieron un índice de 61,7 (48,1 jóvenes y 13,6 adultos mayores), que descendió desde 1963 hasta 2005 (Gráfico 6). En 2018, esta cifra fue de 53,7, con una relación entre adultos mayores y jóvenes cercana a 1:1 (27,4 jóvenes y 26,3 adultos mayores). 


\section{Gráfico 5. América Latina y el Caribe y países miembros de la OCDE: \\ Índice de envejecimiento, 1960 - 2018}

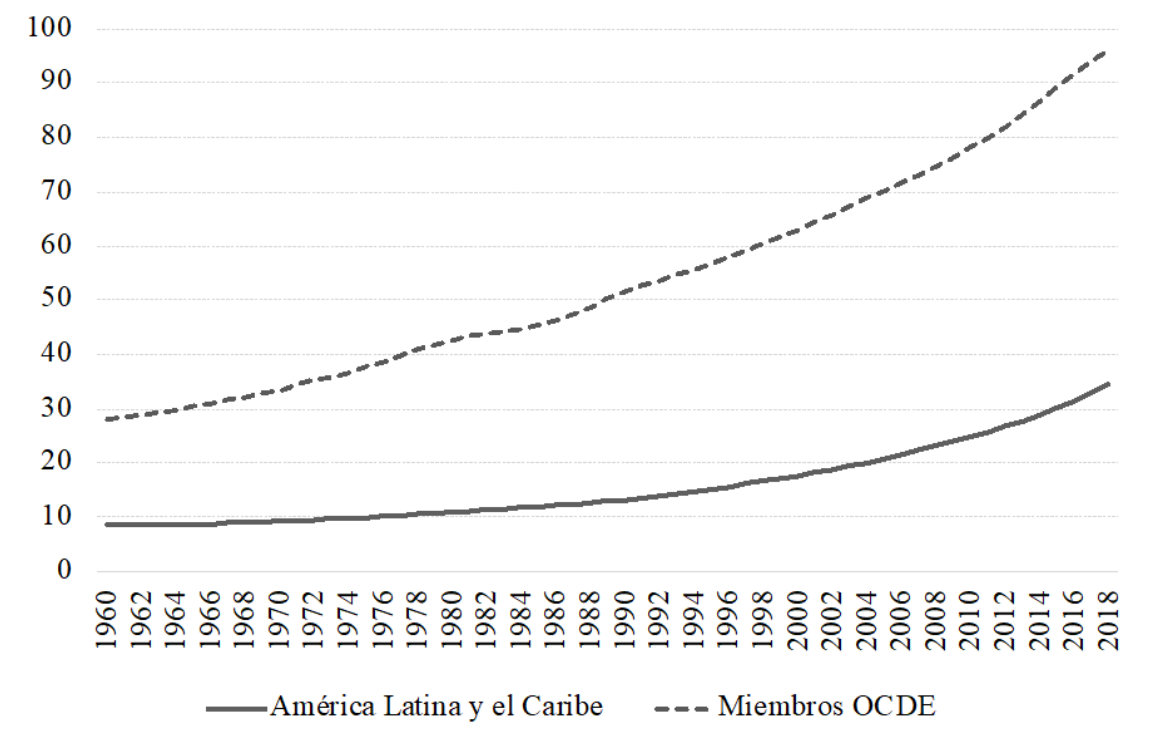

Nota: El índice de envejecimiento expresa la relación entre la cantidad de personas adultas mayores y de niños o jóvenes. El cálculo se realiza como la razón entre el total de personas de 65 años y más sobre las personas menores de 15 años, multiplicado por 100.

Fuente: Elaboración propia con base en datos del Banco Mundial.

\section{Gráfico 6. América Latina y el Caribe y países miembros de la OCDE: Índice de dependencia, 1960 - 2017}

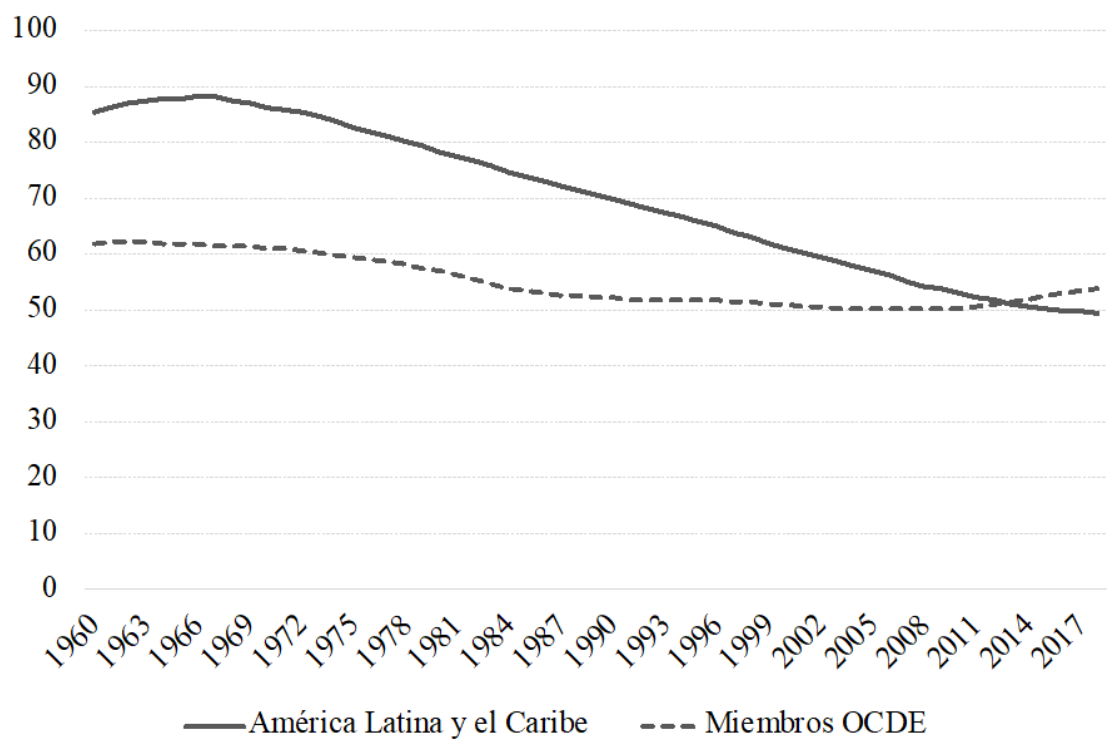

Nota: El índice de dependencia mide la población en edades inactivas como proporción de la población en edades activas o en edad de trabajar. El cálculo se realiza como las personas entre 0 y 14 años (niños y jóvenes) más las personas mayores de 65 años (adultos mayores), todos sobre la población entre 15 y 64 años.

Fuente: Elaboración propia con base en datos del Banco Mundial. 
Cuando avanza el proceso de transición, los países atraviesan un período en el que la población en edad de trabajar crece con respecto a la población dependiente, de forma que disminuye el índice de dependencia. Esto es lo que en la literatura se conoce como "el bono demográfico". Es una oportunidad para incrementar el crecimiento económico y el bienestar de la población en la medida en que disminuye la carga económica sobre las personas potencialmente activas y aumenta la mano de obra disponible (Comisión Económica para América Latina y el Caribe - CEPAL, 2008). Si bien el tiempo del bono demográfico puede durar varias décadas, la relación de dependencia vuelve a aumentar como resultado de la creciente proporción de personas mayores. En América Latina y el Caribe el bono demográfico aún sigue vigente, pero su aprovechamiento depende de un entorno favorable para el empleo, el ahorro, la inversión y la educación. Además, según las estimaciones de la CEPAL, el bono demográfico fue responsable de un incremento del $6 \%$ de los ingresos laborales por consumidores entre 1998 y 2008 de esta región. En algunos países como México y Nicaragua el incremento fue de entre 8 \% y 10 \%; mientras que en otros países como Cuba y Chile fue mucho menor (entre $1 \%$ y 3 \%) (CEPAL, 2008).

\section{TRANSICIÓN DEMOGRÁFICA EN COLOMBIA}

Aunque es evidente que América Latina y el Caribe se encuentra en un proceso de transición demográfica, la velocidad con la que cada uno de los países ha avanzado en las distintas etapas dista de la

del resto. Cuba y Brasil, por ejemplo, se encuentran en etapas avanzadas de la transición, con un crecimiento poblacional por debajo del $1 \%$ desde hace más de una década; y una tasa de fecundidad por debajo del nivel de reemplazo - desde 2005 en Brasil y desde 1980 en Cuba. A diferencia de Cuba, el progreso de Brasil respecto del aumento de la longevidad ha sido más lento que la disminución de la fertilidad. Otros países de América Latina, como Perú, Ecuador y Bolivia, tienen niveles de crecimiento poblacional cercanos al $2 \%$ y tasas de fecundidad por encima del nivel de reemplazo. Colombia, con un crecimiento poblacional de $1,5 \%$ y una tasa de fecundidad por debajo del nivel de reemplazo (1,8 niños por mujer), ha avanzado a una velocidad intermedia en la transición demográfica comparada con la de otros países.

El siglo XIX en Colombia fue de escasos avances desde el punto de vista del progreso económico y social. Solo hasta las últimas décadas empezaron a mejorar algunos de los indicadores demográficos y económicos. El crecimiento de la población fue más bien estable, y la distribución por edades, la tasa de natalidad y la tasa de mortalidad también fueron constantes a lo largo del siglo. Flórez y Romero (2010) utilizan la información de censos, anuarios y memorias para reconstruir la dinámica demográfica del siglo XIX. Las autoras estiman que alrededor de 1800, la población colombiana era de aproximadamente 821.600 personas y para finales de siglo se estima en 4.172.000. La mejoría en las condiciones socioeconómicas se logró en el siglo XX. Durante los primeros años de aquel siglo hubo un progreso lento de la economía, la nutrición y la 
salud pública, producto de la guerra de los Mil Días (Mejía, et al., 2009). Fue solo hasta mediados de siglo que emergió una clara transformación social.

En Colombia, la esperanza de vida a comienzos del siglo XIX era de 26 años, la tasa de mortalidad infantil de 297 defunciones de niños menores de un año por cada 1.000 nacimientos y, en promedio, cada mujer tenía 8,5 hijos. Para inicios del siglo XX, el panorama cambió: la mortalidad infantil se registró en 186,5 muertes por cada 1.000 nacidos en 1905, la esperanza de vida en 39,5 años y la fecundidad en 6,4 niños por mujer.

El crecimiento económico durante la primera mitad del siglo XX y las mejoras en salubridad, alimentación y avances médicos impulsaron en las décadas siguientes una transformación social sin precedentes (Mejía, et al., 2009). La tasa de fecundidad aumentó a 6,7 hijos por mujer en 1960, la expectativa de vida se registró en 57 años, y entre 1965 y 1970, la mortalidad infantil pasó a 90 defunciones por cada 1.000 niños menores de un año (Flórez-Nieto, 2000). Todo esto se tradujo en una población que se cuadruplicó en la primera mitad de siglo y pasó a ser de 17.484.508 en 1964, según cifras del Departamento Administrativo Nacional de Estadística (DANE).

El crecimiento económico sostenido y acelerado empezó después de la década de 1950 y el país pasaría de la sociedad posmaltusiana a incorporarse en la transición demográfica. De acuerdo con el censo de 1985, el 36,4 \% de la población tenía entre 0 y 14 años, el 22,1 \% entre 15 y 24 años, el 37,5 \% entre 24 y 64 años, y solo el 4 \% más de 65 años (Gráfico 7). Es decir, el país era en esencia un país de jóvenes. En estos años, la disminución de la mortalidad infantil, la masificación de los métodos anticonceptivos y la transformación del rol de la mujer dieron paso a un rápido descenso de la tasa de fecundidad que caería a 3,3 hijos en promedio por mujer en 1985 y a 1,8 en 2017 (Gráfico 8).

Sin embargo, el rápido crecimiento económico y el cambio social no abarcaron desde un principio a toda la sociedad colombiana. La transición se inició entre las personas con niveles de ingresos más altos en las principales ciudades y luego se ha ido extendiendo a toda la población. El descenso de la fecundidad, por tanto, evolucionó a ritmos diferentes según los ingresos y el nivel educativo de la madre. En 1985, las mujeres sin ninguna educación tenían en promedio 4,7 hijos y hacia 2015 disminuyó a 3,9. En contraste, las mujeres con educación superior tuvieron en promedio 1,6 hijos tanto en 1985 como en 2015 (Gráfico 9).

La caída reciente de la fecundidad, principalmente en las mujeres en los niveles más bajos de renta y educación, ha disminuido el ritmo de crecimiento de la población y transformado la estructura por edades, como se observa en el Gráfico 7. Estos cambios tienen importantes consecuencias en el crecimiento económico y social. Entre 1960 y 2017, las personas entre 0 y 14 años aumentaron en términos absolutos 1,5 veces. Sin embargo, en términos relativos pasaron de representar el $47 \%$ del total a $23 \%$ durante el mismo período. En contraste, los adultos de 65 años pasaron de ser el $3 \%$ de la población al $8 \%$. El mayor incremento es para las personas en edad de trabajar, que en los últimos 60 años se han cuadruplicado y 


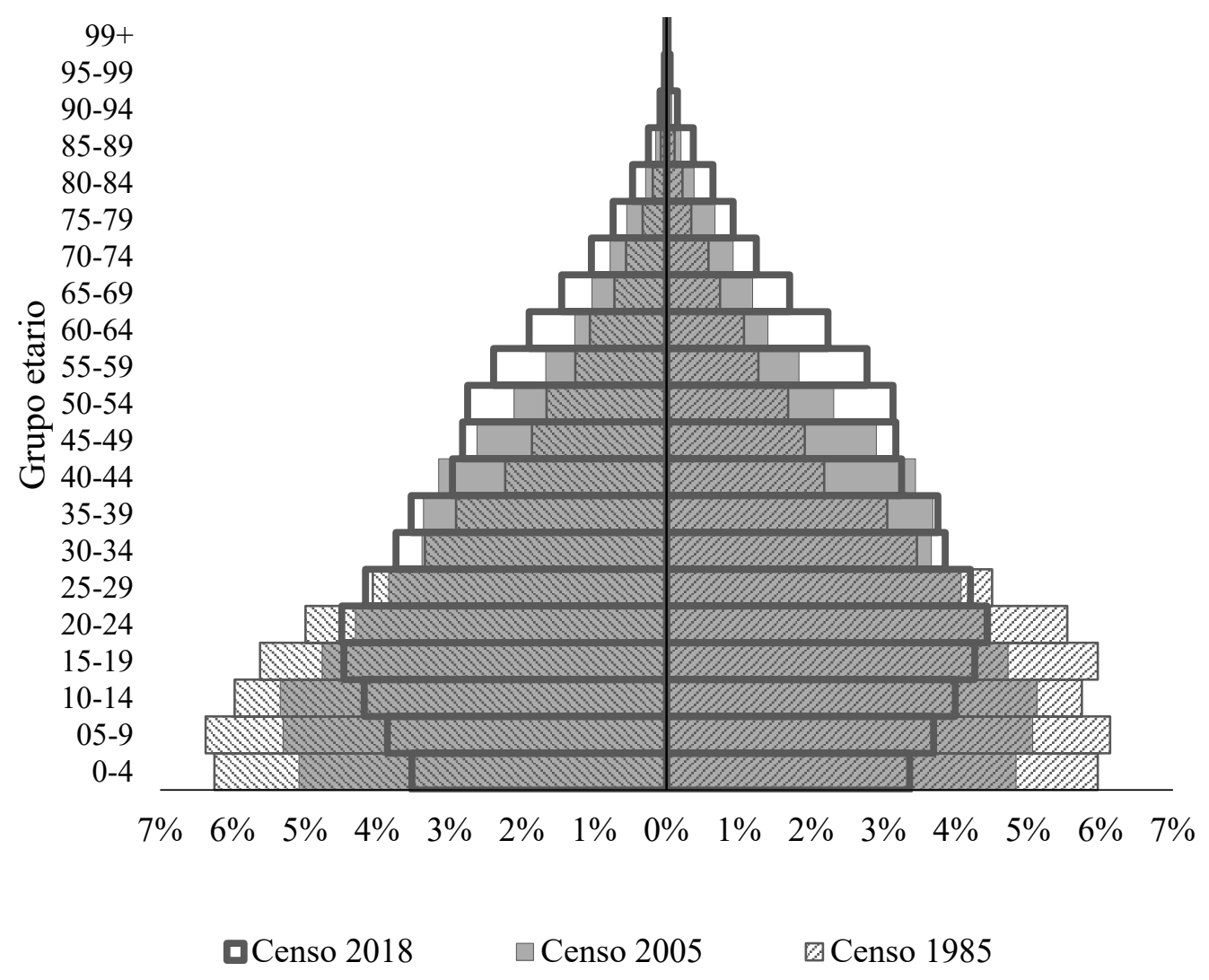

Nota: A la izquierda, hombres; a la derecha, mujeres.

Fuente: Elaboración propia con base en datos del DANE.

pasaron de representar el 50 \% de la población en 1960 a ser el 68 \% en 2017. Sin embargo, este crecimiento en participación es cada vez más lento (Gráfico 10). En este sentido, a partir de 1965 ha disminuido el índice de dependencia (Anexo 1), y se calcula que alcanzará su mínimo en 2027 (Martínez, 2013). Durante este lapso de 57 años, los ingresos por consumidor habrán aumentado en aproximadamente $27 \%$, lo que implicaría un crecimiento anual de los ingresos relativos al consumo de 0,5 \% (Martínez, 2013).

Como se mencionó, no solo hay gran diversidad demográfica entre los países de América Latina, sino también al interior de cada uno de ellos. El proceso de transición demográfica responde en gran medida a las dinámicas sociales y económicas propias de cada país. En Colombia, la evolución de los indicadores sociales difiere entre sus distintas regiones. Las personas de la periferia del país se enfrentan consistentemente a indicadores inferiores en materia de salud, educación y crecimiento económico en relación con el centro del país (Barón 2003; Bonet y Meisel, 1999; Galvis y Meisel, 2012; Meisel y Granger, 2019). 
Gráfico 8. Colombia y América Latina y el Caribe:

Tasa global de fecundidad, 1960 - 2016

(número de hijos promedio por mujer)

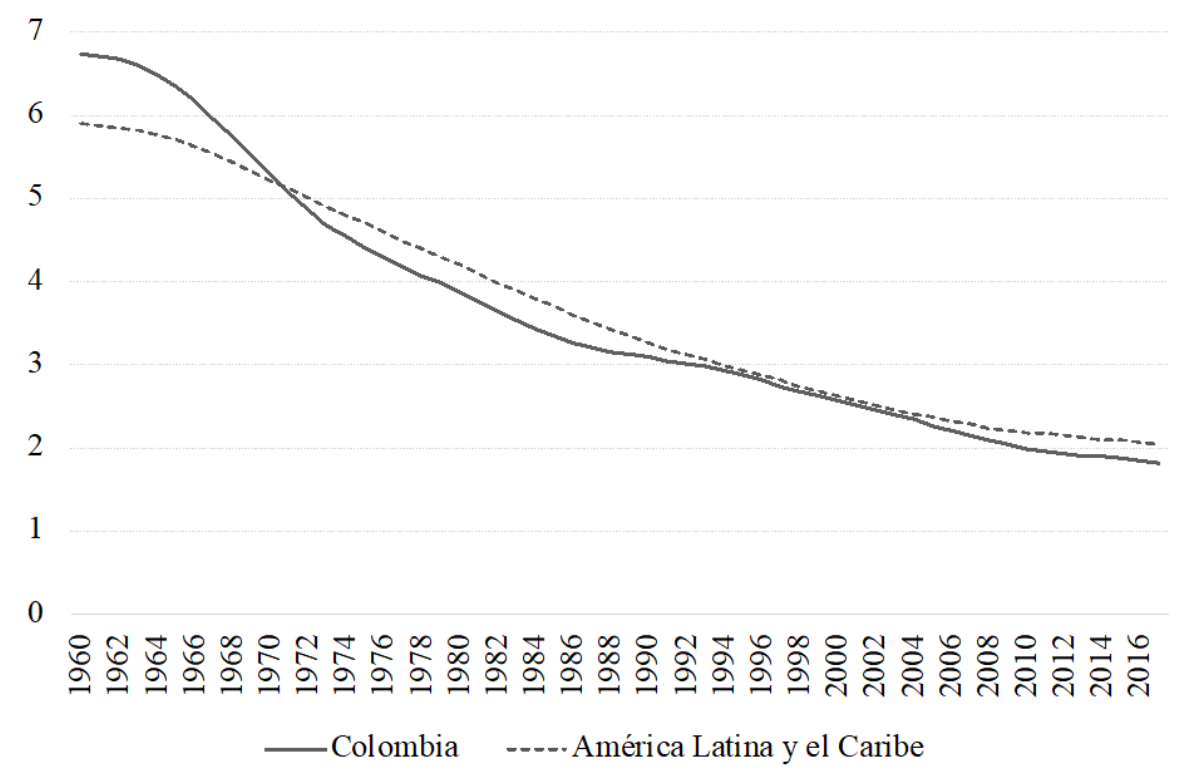

Fuente: Elaboración propia con base en datos del Banco Mundial.

\section{Gráfico 9. Colombia: Tasa global de fecundidad por nivel educativo de la madre, 1985 - 2015 \\ (número de hijos promedio por mujer)}

6

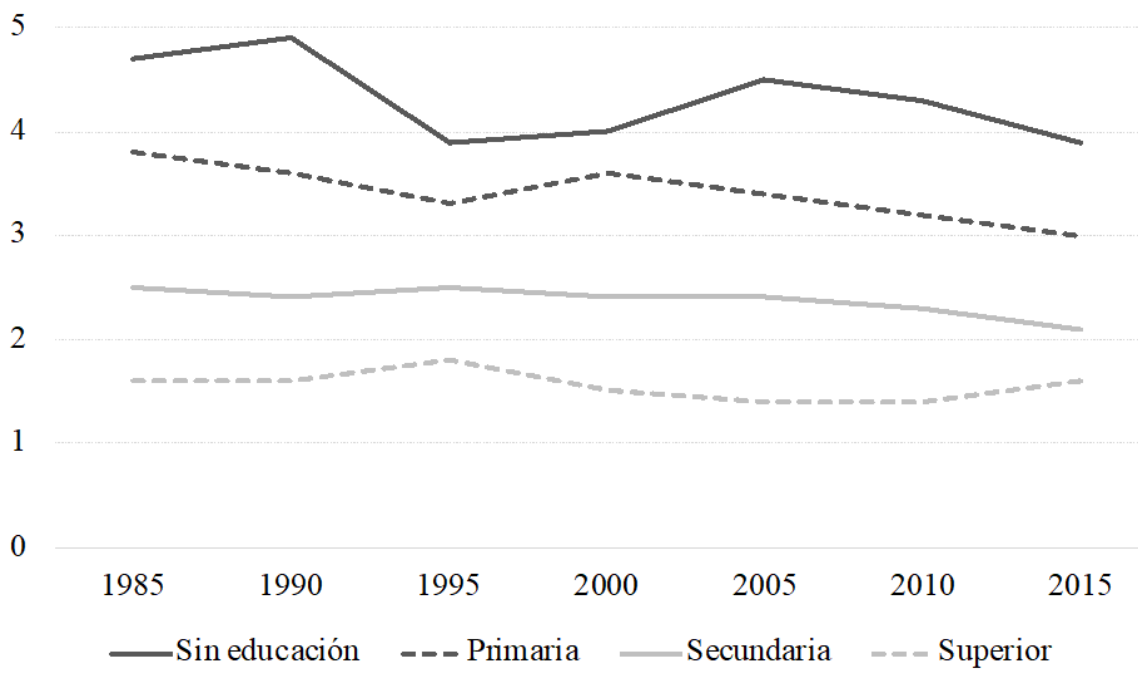

Fuente: Elaboración propia con base en datos de la Encuesta Nacional de Demografía y Salud (ENDS). 


\section{Gráfico 10. Colombia: Evolución de la población por grupos etarios, 1960 - 2017 (número de personas)}

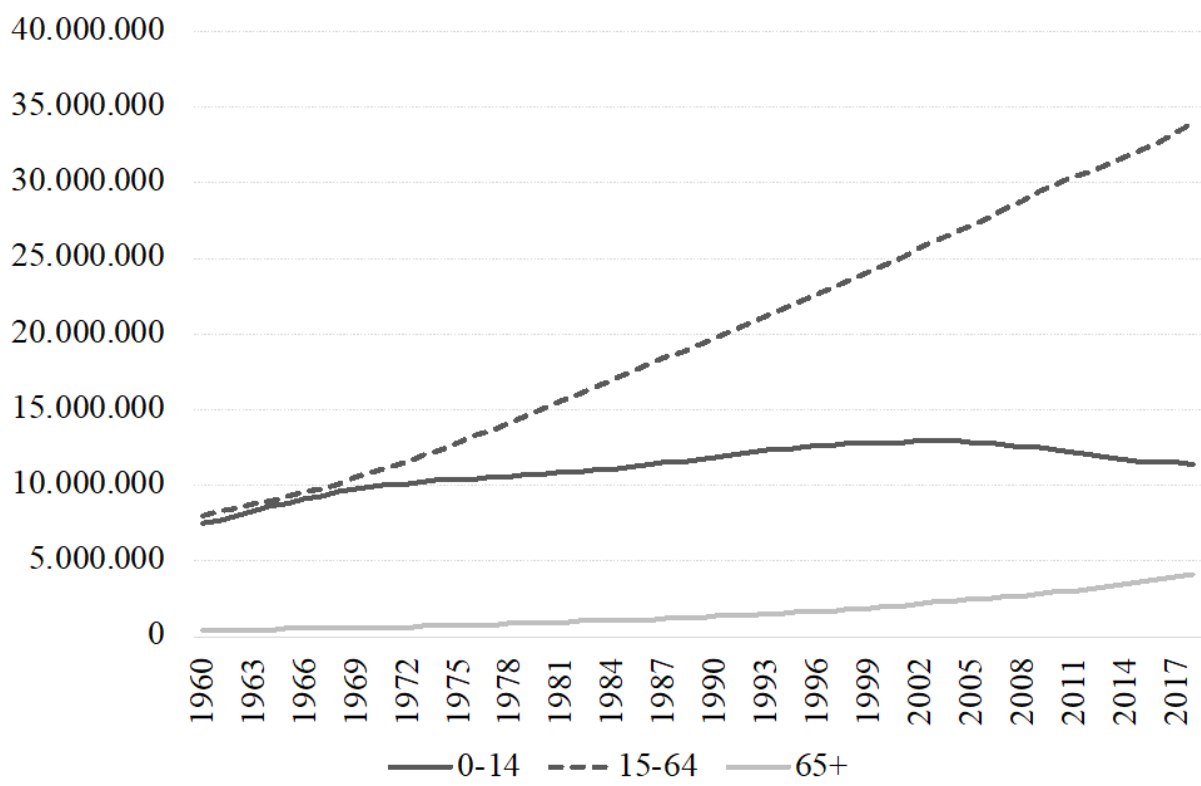

Fuente: Elaboración propia con base en datos del Banco Mundial.

Todas las regiones evidencian desde la segunda mitad del siglo XX un descenso en sus tasas de fecundidad. Sin embargo, la posición relativa entre ellas en materia de fecundidad no ha variado en los últimos 50 años. El mayor descenso se produjo en la región Caribe y en la región Oriental, pues registraron 7,8 y 7,9 hijos promedio por mujer en 1970 y 2,5 y 2,0 en 2015, respectivamente. Para el caso de Bogotá, la fecundidad disminuyó de 4,5 a 1,8 en el mismo período, en la región Central de 6,8 a 1,6 y en el Pacífico de 5,9 a 1,9 hijos por mujer. En lo que respecta al Pacífico, si se consideran solo los municipios litorales, la tasa es la más alta del país, con 2,8 hijos en promedio por mujer en 2015. El decrecimiento ocurrió principalmente entre 1970 y 1995, y a partir de este último año la reducción ha sido más moderada, con una mayor disminución en Bogotá y las regiones Central y Oriental (Gráfico 11). En la actualidad, la región Caribe y el litoral Pacífico son las regiones con mayor fecundidad, por encima del nivel de reemplazo y del promedio nacional, mientras que la región central y Bogotá tienen las tasas más bajas (Gráfico 11).

Durante las últimas décadas, la tendencia decreciente en el número de hijos por mujer y una migración incipiente explica que la población entre 0 y 14 años sea cada vez más pequeña en términos relativos. Entre 1985 y 2018, este grupo etario decreció, sobre todo en las regiones Central y Oriental (13 pp y 15 pp, respectivamente), mientras que en los nuevos departamentos de la Orinoquía y la Amazonía el decrecimiento ha sido más leve. Asimismo, las regiones que han experimentado una mayor reducción en la población joven como porcentaje del total de la población experimentan también un mayor aumento de la participación de la población en edad de trabajar y de la mayor de 64 años (Gráficos 12, 13, 14 y 15). 
Gráfico 11. Colombia: Tasa global de fecundidad por regiones, 1970 - 2015 (número de hijos promedio por mujer)

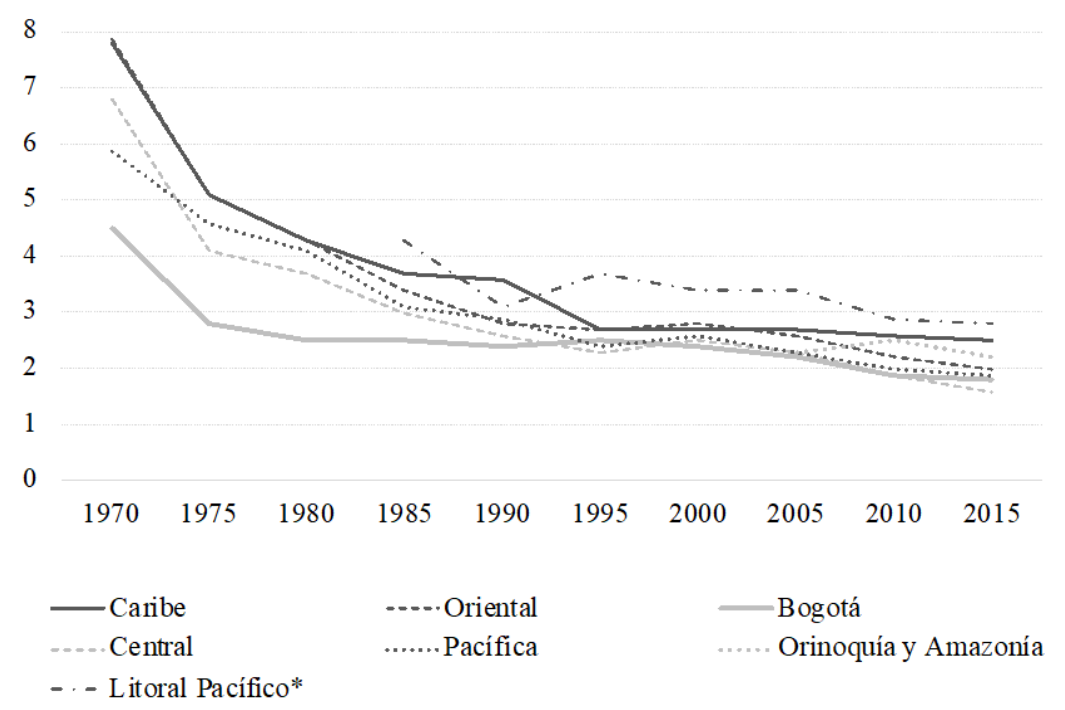

Notas: (1) Región Caribe: La Guajira, Cesar, Magdalena, Atlántico, San Andrés, Bolívar, Sucre y Córdoba. Región Oriental: Santanderes, Boyacá, Cundinamarca y Meta. Región Central: Antioquia, Caldas, Risaralda, Quindío, Tolima, Huila y Caquetá. Costa Pacífica: Valle del Cauca, Cauca, Nariño y Chocó. Orinoquía y Amazonía: Arauca, Casanare, Guainía, Vichada, Amazonas, Putumayo, Guaviare y Vaupés. (2) El litoral Pacífico incluye al Chocó, Cauca y Nariño entre 1985-1995, y desde 2000 solo los municipios limítrofes con el océano Pacífico. Esto último se debe a un cambio en la metodología de la ENDS. (3) Los datos de la ENDS calculan la tasa global de fecundidad como el promedio de hijos por mujer en los últimos tres años antes de la encuesta.

Fuente: Elaboración propia con base en datos de la ENDS.

\section{Gráfico 12. Colombia: Evolución de la población entre 0 y 14 años como porcentaje de la población total por regiones, 1985 y 2018}

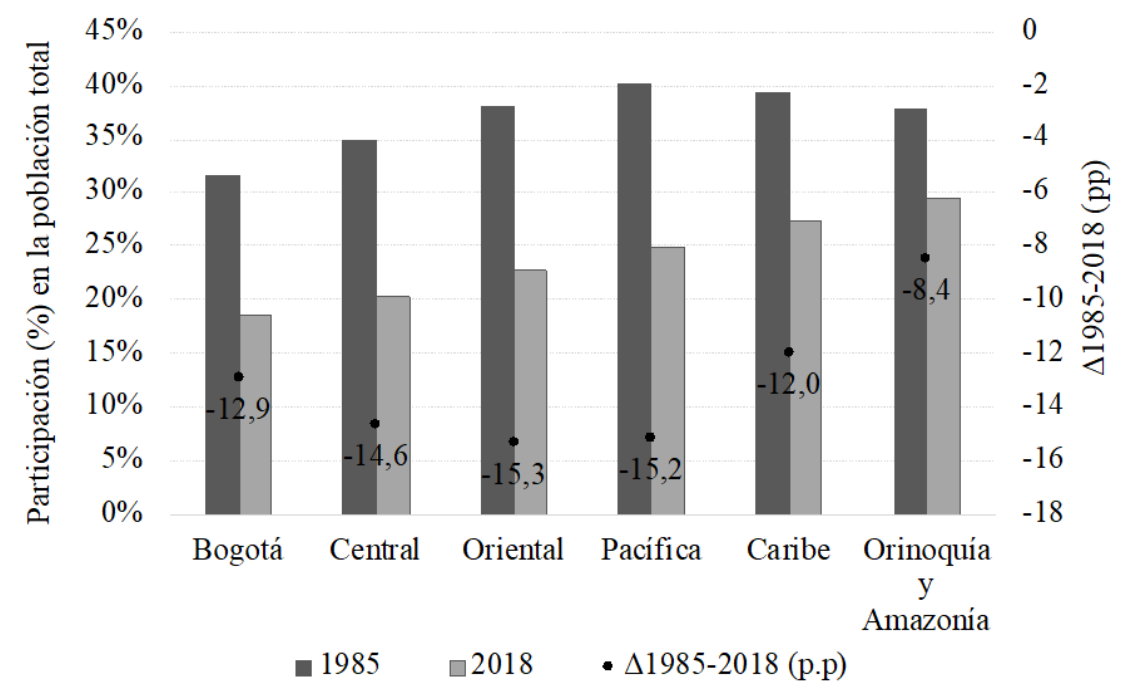

Fuente: Elaboración propia con base en datos censales del DANE. 
Gráfico 13. Colombia: Evolución de la población entre 15 y 24 años como porcentaje de la población total por regiones, 1985 y 2018

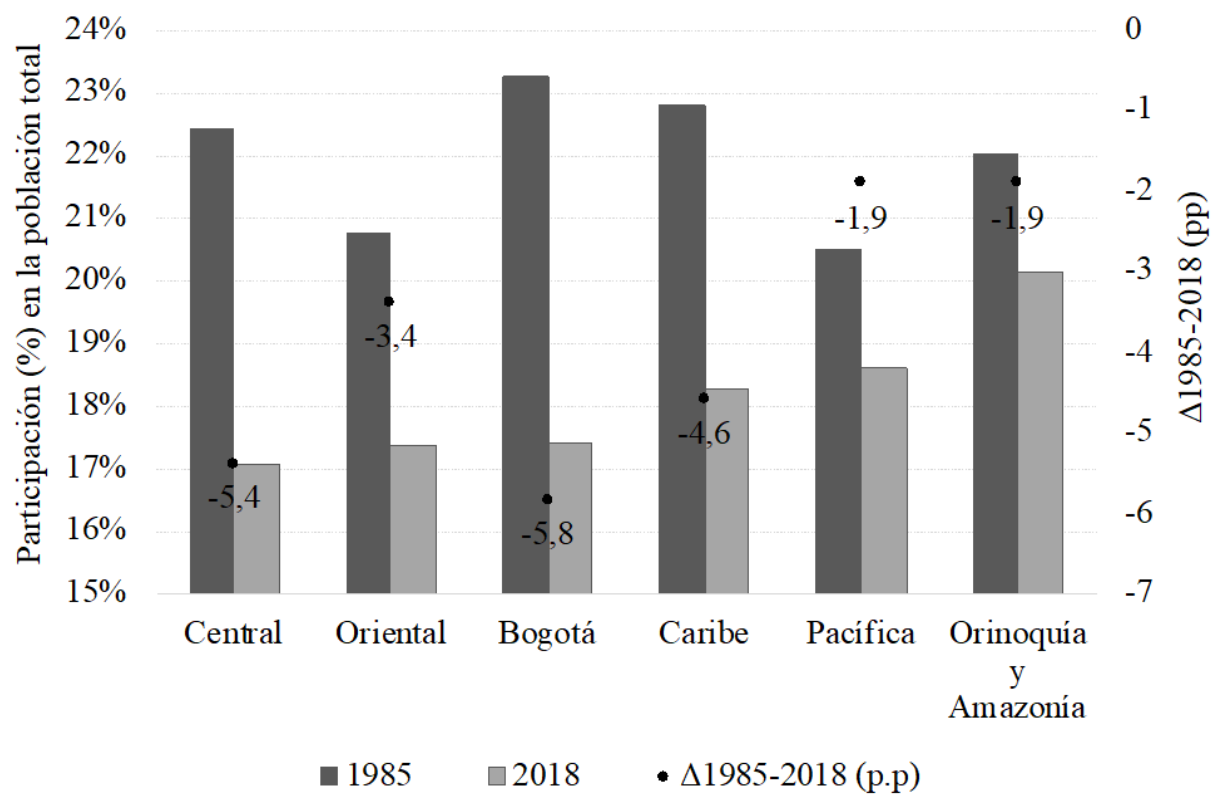

Fuente: Elaboración propia con base en datos censales del DANE.

Gráfico 14. Colombia: Evolución de la población entre 15 y 64 años como porcentaje de la población total por regiones, 1985 y 2018

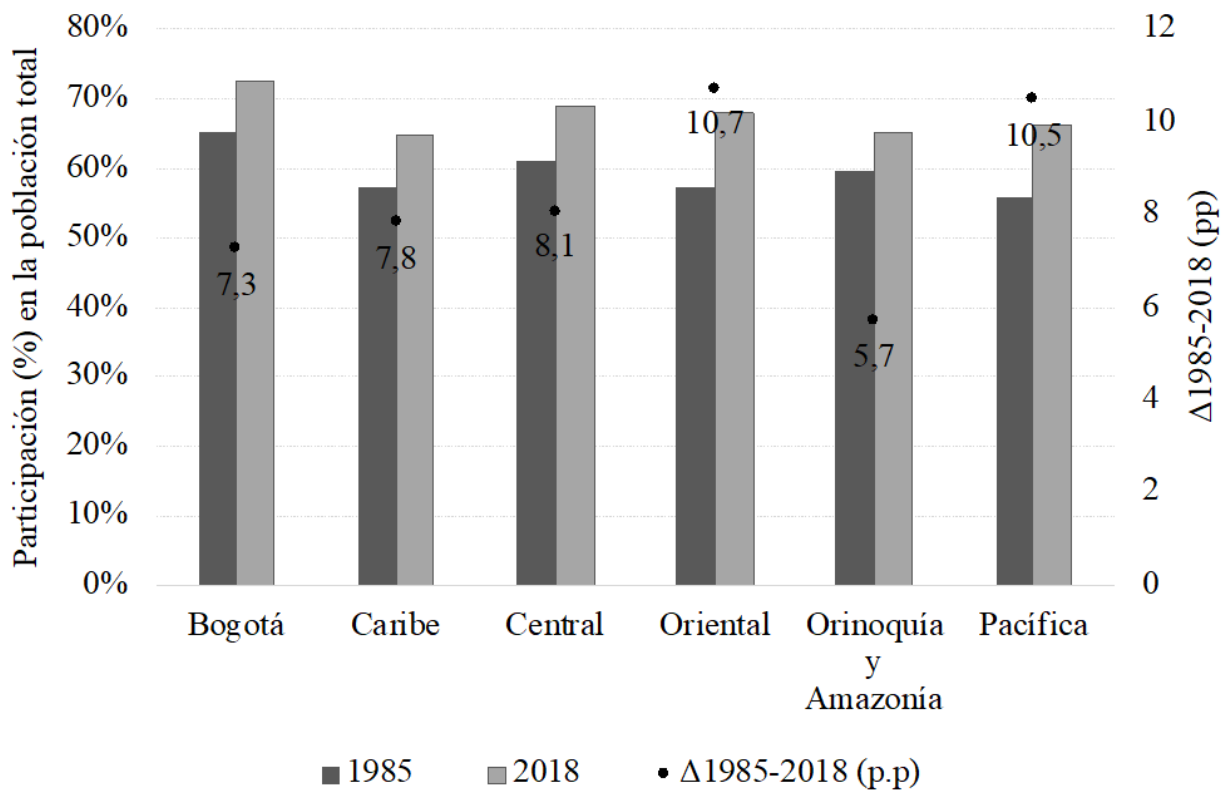

Fuente: Elaboración propia con base en datos censales del DANE. 
Gráfico 15. Colombia: Evolución de la población de 65 años o más como porcentaje de la población total por regiones, 1985 y 2018

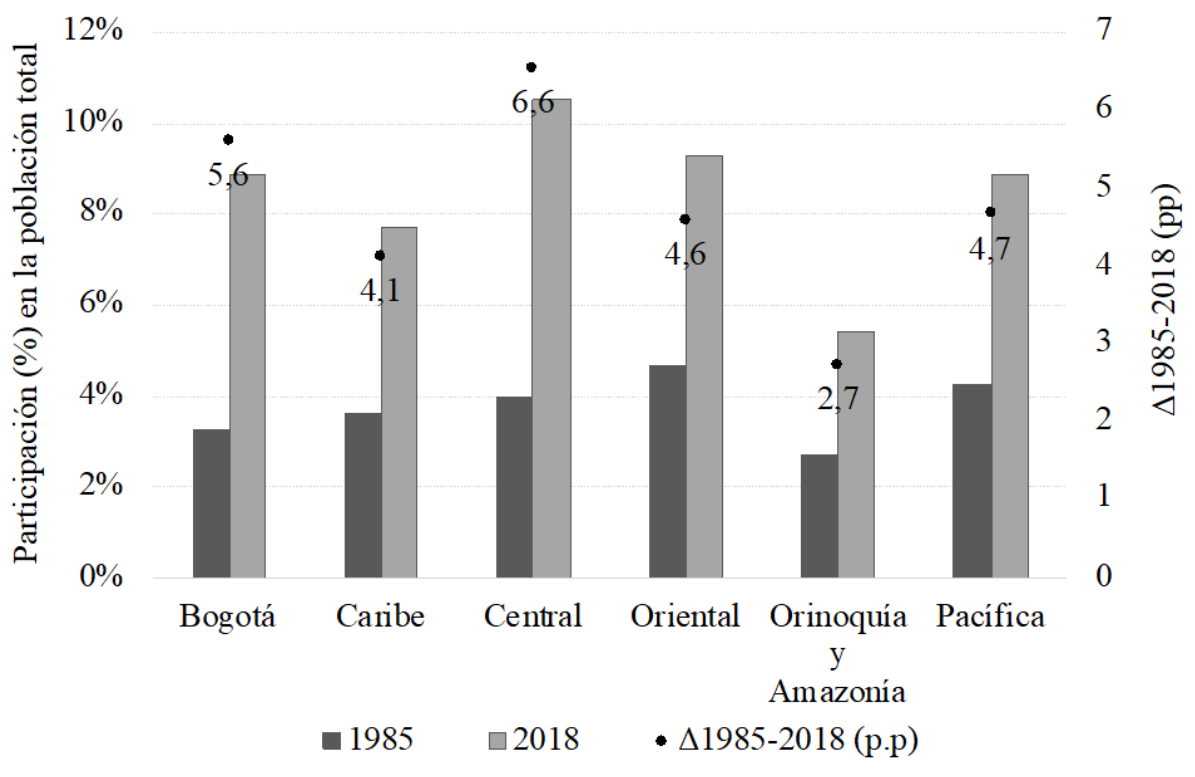

Fuente: Elaboración propia con base en datos censales del DANE.

En este sentido, el decrecimiento de la fecundidad es uno de los principales cambios sociales de la segunda mitad del siglo XX. A pesar de las disparidades regionales, en Colombia esta transición hacia bajas tasas de fecundidad y una mayor esperanza de vida es un hecho generalizado. No obstante, las regiones empezaron desde distintos puntos de partida y esas brechas en el proceso de transición no han logrado desaparecer. Las regiones del centro del país lideran la transición, probablemente producto de un mayor progreso social y económico, y unos pasos atrás siguen las regiones de la periferia. En todos los casos, dicha reestructuración de la composición por edad de la población colombiana acarrea nuevos retos para suplir las necesidades de una población cada vez más envejecida y con una fuerza laboral de menor tamaño.

En síntesis:

- Colombia, al igual que la mayoría de los países de América Latina y el Caribe, inició en el siglo XX un proceso acelerado de transformación social que dio lugar a nuevas dinámicas demográficas que se acentuaron después de 1950.

- Durante el siglo XX, la población en Colombia pasó de cerca de 5 millones a 40 millones de habitantes. En este período, la esperanza de vida aumentó de 39,5 a 73 años. Durante los últimos cincuenta años, la tasa de fecundidad pasó de ser 6,7 en 1960 a 1,8 en 2017, y los jóvenes entre 0 y 14 años pasaron de ser el $47 \%$ de la población total a representar el $23 \%$. 
- Colombia, al igual que Venezuela, México y República Dominicana, se encuentra en una etapa avanzada de la transición demográfica. En contraste, otros países como Brasil, Chile, Cuba y Argentina alcanzaron los niveles de reemplazo una o varias décadas antes que Colombia. Otros países, como Perú, Bolivia, Ecuador, Haití y El Salvador, se encuentran todavía en una etapa inicial de la transición.

- Desde 1970 hasta la actualidad, Colombia ha experimentado un aumento de la población en edad de trabajar y, por tanto, de la fuerza laboral potencial. Esto, que en la literatura se conoce como el bono demográfico, ha sido una oportunidad que está próxima a cerrarse. El país enfrentará en las siguientes décadas los retos que supone el envejecimiento de la población.

- La disminución de los niveles de fecundidad está relacionada con los niveles de ingreso y educación de las mujeres. En un país como Colombia se relaciona también con el origen regional. Todas las regiones han reducido la tasa de fertilidad desde 1970, pero la región Caribe y el litoral Pacífico conservan mayores tasas de fecundidad, reflejo del rezago de la periferia en el progreso social y económico nacional.

\section{EFECTOS DE LA TRANSICIÓN DEMOGRÁFICA EN LA POBLACIÓN UNIVERSITARIA}

\section{A. Una aproximación a la demanda potencial}

Uno de los desafíos que impone la transición demográfica es la reducción de la demanda por educación. Dada la menor fecundidad, el número de niños nacidos cada año disminuye y, con ello, también lo hace el tamaño de las cohortes que ingresan a la etapa escolar y posteriormente a la universidad.

En Colombia no hubo grandes logros en materia educativa durante la primera mitad del siglo XX, a pesar del progreso social y material de aquella época. La matrícula en educación primaria como porcentaje de la población total se mantuvo en alrededor de $6 \%$ a nivel nacional con grandes brechas regionales: el 6,7 \% en Antioquia, el 4,1 \% en el Tolima, el 3,3 \% en el Huila, el 2,3 \% en Boyacá, el 1,8 \% en Santander, el 1,9 \% en Bolívar y el 1,2 \% en Atlántico (Ramírez-Giraldo y Téllez-Corredor, 2006). Solo hasta la década de 1950 empezaron a mejorar significativamente los indicadores educativos en el país. La tasa de escolaridad bruta en educación secundaria, por ejemplo, subió del 6,2 \% en 1951 hasta aproximadamente el 70 \% en el 2000. Además, el número de años promedio de educación en zonas urbanas pasó de 4,2 en 1951 a 8,3 en los inicios del nuevo siglo.

Las tasas de fecundidad en Colombia, como en el mundo, han estado estrechamente relacionadas con la evolución de la educación. Puesto que los individuos acceden a ella desde los primeros años de vida, la educación contribuye a formar las conductas relacionadas con la sexualidad, el matrimonio, la fecundidad, el cuidado personal, entre otras actitudes y hábitos. Por lo tanto, en la medida que progresó la cobertura 
educativa en Colombia, disminuyó también la tasa de fecundidad. Y, paralelamente, el crecimiento de la cobertura en educación compensó, por un tiempo, el efecto negativo de una tasa de fecundidad cada vez más baja sobre el tamaño de las cohortes de estudiantes. Sin embargo, en lo corrido del siglo XXI, con grandes avances en la población atendida, la transición demográfica empezó a reflejarse en la demanda educativa (Gráfico 16).

El Gráfico 16 muestra el crecimiento indexado del total de estudiantes entre 14 y 21 años, según año de nacimiento, que presentaron las pruebas Saber 11 en algún momento entre 2000 y 2018. Esto, como aproximación de las cohortes de estudiantes que culminan bachillerato y que constituyen la demanda potencial por educación superior. Como se observa, el tamaño de las cohortes de 1984 en adelante se mantuvo en aumento hasta 1996, año a partir del cual empieza a decrecer. A pesar de que es un fenómeno reciente, esta información sugiere que a partir de 1996 las cohortes de estudiantes que culminan el colegio son cada vez más pequeñas.

\section{Gráfico 16. Colombia: Estudiantes entre 14 y 21 años que presentaron las pruebas Saber 11 entre 2000 y 2018 por año de nacimiento y región (crecimiento indexado: $1984=100$ )}

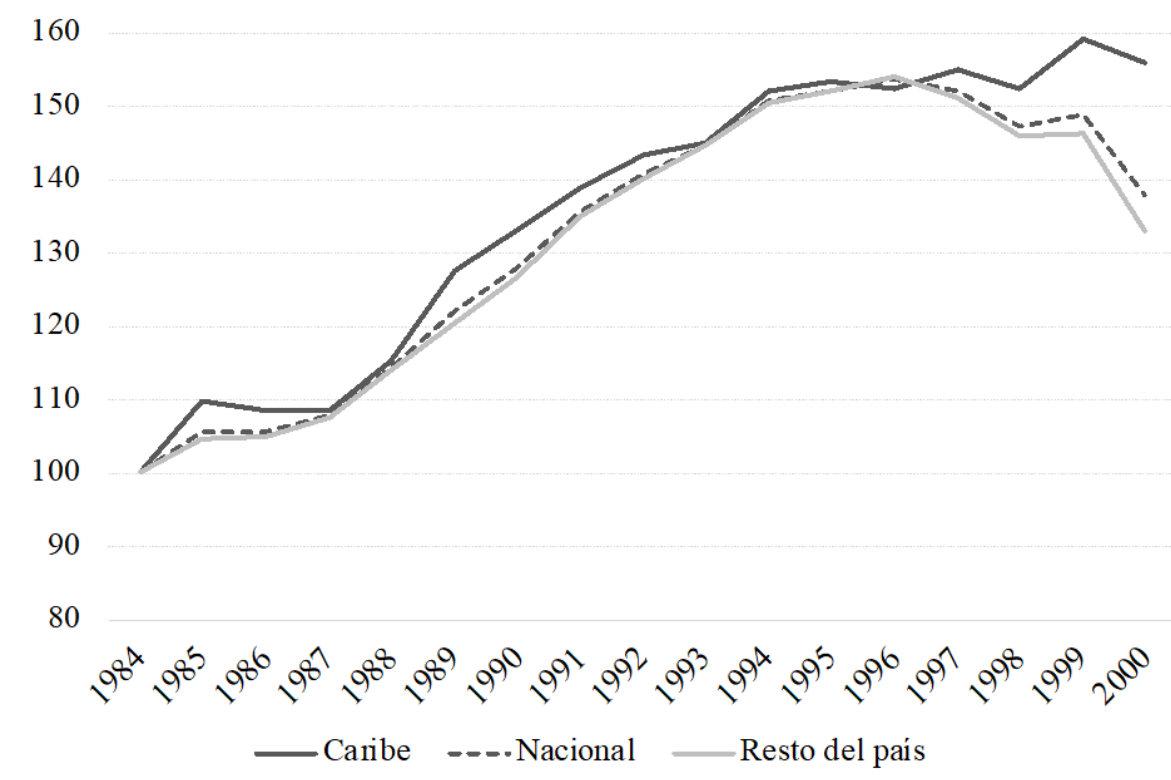

Nota: El 93,6\% de los estudiantes que presentan la prueba Saber 11 entre 2000 y 2018 tiene entre 14 y 21 años, esto significa que nacieron entre 1979 y 2004. Sin embargo, el Gráfico se restringe a las cohortes entre 1984 y 2000 debido a que en los años anteriores o posteriores hay mayor cantidad de estudiantes que presentaron la prueba Saber 11 ya sea antes del 2000 (aquellos nacidos antes de 1984) o después de 2018 (nacido después de 1997) que quedan por fuera. Es decir, el tamaño de las cohortes en estos años estaría sesgado hacia abajo.

Fuente: Elaboración propia con base en datos del Instituto Colombiano para la Evaluación de la Educación (Icfes). 
El análisis puede estar sesgado por la exclusión de los estudiantes entre 19 y 21 años que nacieron después de 1997 y que hasta 2018 no habían presentado la prueba Saber 11. En la cohorte de 1998, solo hay estudiantes entre 14 y 20 años, en 1999 entre 14 y 19 años y en 2000 entre 14 y 18 años. Por tanto, la disminución observada en el tamaño de la cohorte podría ser el resultado de un grupo de edad más acotado que en los otros años. Para comprobar que esto no altera las conclusiones, realizamos el mismo análisis solo para estudiantes entre 14 y 18 años (que son el 83 \% de los estudiantes que presentan la prueba Saber 11). En este caso, el grupo de edad es igual para todas las cohortes y las tendencias son muy similares (Anexo 2).

De nuevo, el descenso de la demanda potencial por educación no es homogéneo a lo largo del país. Como se observa en el Gráfico 16, en la región Caribe el crecimiento está por encima del promedio nacional. Además, si se desagrega por las principales áreas metropolitanas, el crecimiento del número de estudiantes por cohorte de nacimiento - que representa la demanda potencial por educación universitaria - fue más acelerado en Bogotá y Medellín durante los primeros años, mientras que Barranquilla fue la de menor crecimiento. Sin embargo, contrario al resto de las áreas metropolitanas, en esta última ciudad las cifras no disminuyen a partir de 1996 (Gráfico 17).

Gráfico 17. Colombia: Estudiantes entre 14 y 21 años que presentaron las pruebas Saber 11 entre 2000 y 2018 por año de nacimiento y principales áreas metropolitanas (Crecimiento indexado: $1984=100)$

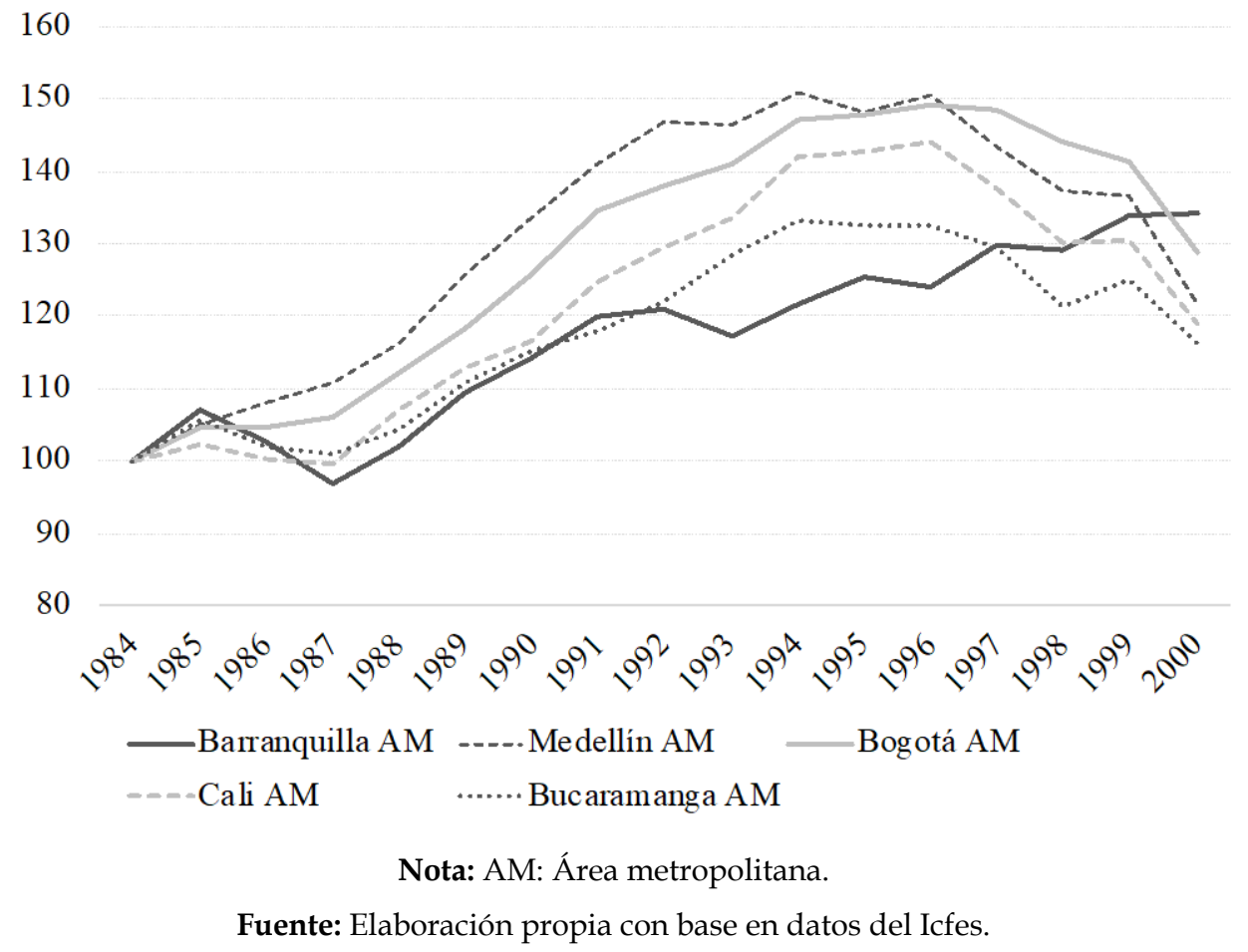




\section{B. Heterogeneidad regional: El caso del Caribe colombiano}

El comportamiento del número de estudiantes que presentaron las pruebas Saber 11 entre 2000 y 2018 indica que el crecimiento del tamaño de las cohortes en la región Caribe es mayor que el del total nacional. La explicación a este hecho puede estar relacionada con el acceso y la eficiencia de la educación en la región, o bien, con las tendencias demográficas del Caribe que difieren de las del resto del país. A continuación, se analizan indicadores relacionados con cada uno de estos aspectos con el fin de identificar cuáles de estas hipótesis explican mejor esta tendencia.

\section{La cobertura en educación}

El tamaño de las cohortes de estudiantes que finalizan la educación media es en parte definido por políticas que determinan el acceso a la educación en cada uno de los niveles. En Colombia, la cobertura en educación primaria es en gran medida universal, pues alrededor del $90 \%$ de los estudiantes de entre 5 y 10 años están matriculados en primaria. Sin embargo, el reto permanece en el acceso a preescolar, secundaria y media. Dado que nuestro análisis se basa en los estudiantes que presentan las pruebas Saber 11, nos enfocaremos en los últimos niveles educativos, secundaria y media.

Como se observa en el Gráfico 18, la cobertura en educación secundaria y media en la región Caribe estuvo por debajo, aproximadamente en 2 pp, del nivel nacional entre 2007 y 2015. Las tendencias tanto regional como nacional fueron crecientes hasta 2013, momento en el que se observa un descenso de la cobertura, que luego se recupera en 2016. En los últimos tres años, el crecimiento de la región Caribe ha sido mayor al nacional, lo que permitió que la cobertura en educación secundaria y media, tanto en el Caribe como a nivel nacional, se ubicara alrededor del 82 \% en 2018.

Esta información sugiere que, a pesar de que la cobertura en la región Caribe fue más baja que el promedio nacional durante buena parte del período evaluado (Gráfico 18), las cohortes que ingresaron al colegio y posteriormente presentaron la prueba Saber 11 en su último año educativo crecieron por encima del resto del país (Gráfico 16). Adicionalmente, la evolución de la cobertura sigue la tendencia nacional, excepto en los últimos tres años, que es aún muy reciente para atribuirle el comportamiento observado entre 2000 y 2018.

\section{Eficiencia de la educación}

El número de estudiantes que finaliza la educación básica y media no es sólo una función de la cobertura sino también de la eficiencia de la educación, es decir, de la capacidad del sistema educativo para retener la población matriculada hasta que finalice el ciclo de estudios. 
Mayores avances en indicadores de deserción estudiantil y repitencia podrían explicar que cada vez más estudiantes finalicen su educación media. Este es precisamente el caso de la región Caribe, que entre 2011 y 2018 redujo su tasa de deserción en educación secundaria y media en 1,7 pp y 2,1 pp, respectivamente; y se ubicó por debajo de las cifras a nivel nacional (Gráfico 19).

\section{Gráfico 18. Colombia: Cobertura neta en educación} secundaria y media por regiones, 2007 - 2018

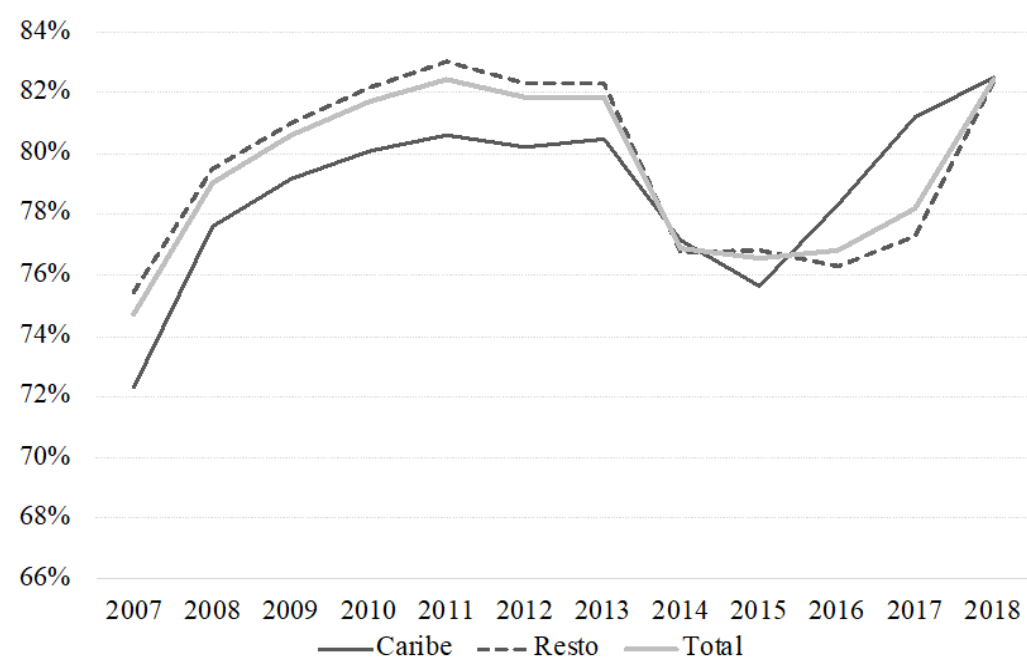

Nota: Los cálculos de cobertura se realizan como la proporción de estudiantes entre 11 y 16 años matriculados en educación media y secundaria. Se utilizan las proyecciones del censo de 2005 y los datos de matrícula del DANE.

Fuente: Elaboración propia con base en datos del DANE.

\section{Gráfico 19. Colombia y región Caribe: Tasa de deserción estudiantil en educación secundaria y media, 2011 y 2018 (porcentajes)}

a. Secundaria

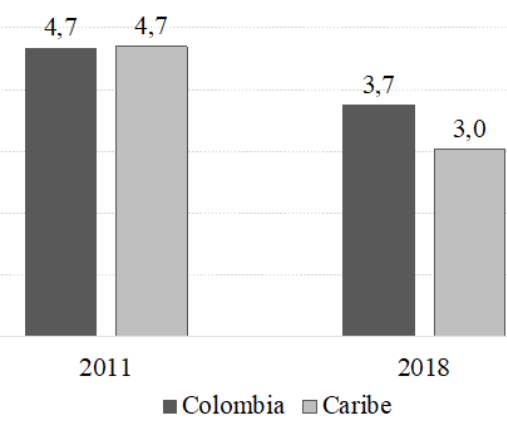

b. Media

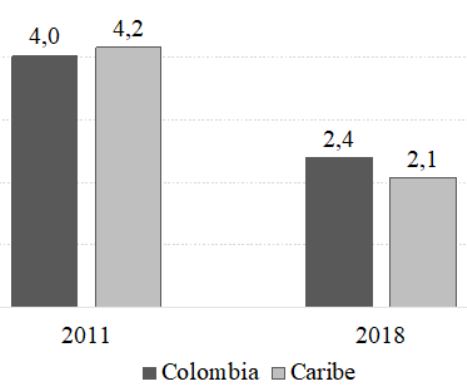

Nota: La tasa de deserción de la región Caribe se calcula como un promedio ponderado por la participación en los estudiantes matriculados en cada nivel educativo.

Fuente: Elaboración propia con base en datos del Ministerio de Educación. 
Aunque las limitaciones en los niveles de desagregación de los datos impiden hacer este cálculo para todo el período, es posible afirmar que la evolución de las cohortes que se observa en el Gráfico 16 podría responder a mayores avances en la eficiencia del sistema educativo en la región Caribe en relación con el promedio nacional. No obstante, el poder explicativo es bajo. Las diferencias entre la disminución de la tasa de deserción nacional y del Caribe en los últimos siete años son menores a 1 pp, mientras que la brecha en el crecimiento indexado de las cohortes de estudiantes que presentaron la prueba Saber 11 entre 2000 y 2018 es de 4 pp en promedio cada año. ${ }^{2}$ Además, en el resto del país el tamaño de las cohortes ha disminuido desde 1996 a pesar de que la deserción también lo ha hecho.

\section{Tendencias demográficas}

Existe una causa adicional a las políticas educativas que ayuda a explicar el comportamiento observado en las cohortes de graduados del colegio. En la sección anterior se muestra que la tasa de fecundidad en la región Caribe, en conjunto con la del litoral Pacífico, ha sido la más alta a nivel nacional. En 2015, las mujeres del Caribe tenían en promedio 2,5 hijos, equivalente a la tasa de fecundidad nacional en 1990. En contraste, ese mismo año en Colombia se registraron 1,8 hijos por mujer. Esta tendencia de nacimientos explica por qué mientras la población entre 11 y 16 años (en edad de estudiar secundaria y media) decrecía en el resto del país, en la región Caribe siguió creciendo entre 2007 y 2017, y luego decreció ligeramente en 2018. En particular, en la región Caribe el tamaño de la población entre 11 y 16 años disminuyó 2 \% entre 2007 y 2018 (principalmente por la caída en los últimos dos años), mientras que en el resto del país la cifra fue de $15 \%$ (Gráfico 20).

El patrón regional en el crecimiento de las cohortes de estudiantes que presentan la prueba Saber 11 por año de nacimiento (observado en el Gráfico 16) es explicado principalmente por diferencias en las tendencias demográficas entre la periferia y el centro del país. Aunque los avances en eficiencia y acceso a la educación pueden ser relevantes, sus efectos sobre el tamaño de las cohortes son limitados y más recientes.

Por lo anterior, se derivan dos conclusiones preliminares: 1) las cohortes de estudiantes por año de nacimiento crecieron desde 1984 hasta 1996 a nivel nacional, y en el Caribe crecieron por encima del promedio nacional y continuaron haciéndolo después de 1996; 2) la causa más plausible de este comportamiento heterogéneo se atribuye a factores demográficos.

${ }^{2}$ No hay información disponible sobre la tasa de deserción departamental entre 2000 y 2011, por lo que no es posible estimar la deserción regional en ese período. 
Gráfico 20. Colombia: Población entre 11 y 16 años, 2007 - 2018

(crecimiento indexado: $2007=100$ )

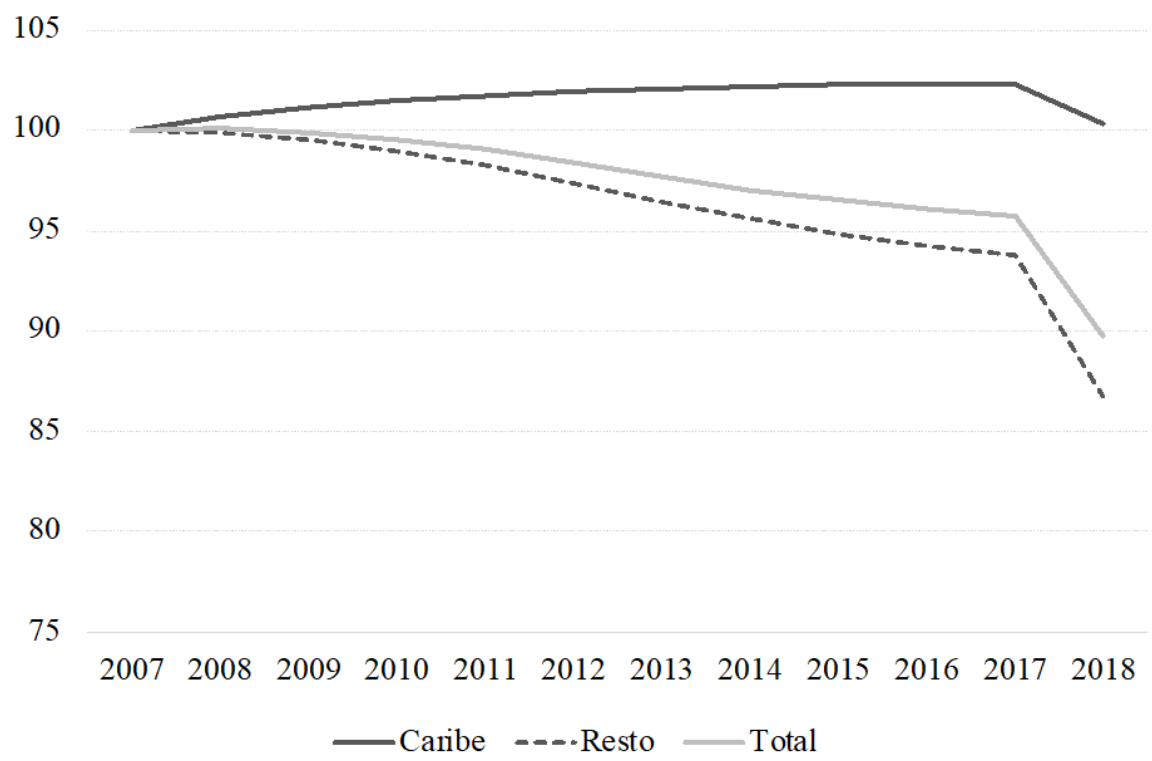

Fuente: Elaboración propia con base en datos del DANE.

\section{Demanda efectiva por educación superior}

La demanda efectiva por educación superior se mide por las inscripciones en instituciones educativas, que se mantuvo con una tendencia creciente entre 2000 y 2016. Ese es el caso tanto en instituciones públicas como privadas. A partir de 2017, Colombia experimenta una disminución en el número de inscripciones en instituciones privadas y en 2018 en las públicas (Gráfico 21). Para el caso de las universidades privadas, las inscripciones cayeron $4 \%$ y $14 \%$ en los últimos dos años, y en las universidades públicas el crecimiento fue de $13 \%$ y $-2 \%$. Estas cifras contrastan con los valores promedio: entre 2000 y 2016, las inscripciones crecieron $4 \%$ en las universidades públicas y $8 \%$ en las universidades privadas (Gráfico 22).

Dado que se observa una caída tanto en las inscripciones en universidades como en instituciones de educación superior (IES) en general, el efecto observado no obedece a un desplazamiento de la demanda hacia otros niveles de formación (técnica o tecnológica, por ejemplo). Si se examina el crecimiento indexado de las inscripciones y la matrícula en primer curso de cada uno de los niveles de formación entre 2000 y 2018, se observa un decrecimiento en la mayoría de ellos en los últimos dos años (Anexos 3 y 4). Sin embargo, en este documento el enfoque reposa sobre el caso de las universidades.

Por regiones, nuevamente las inscripciones en el último año disminuyeron en todos los casos, excepto en la región Caribe. El mayor decrecimiento se registró en Bogotá, con una disminución de 40.417 inscripciones menos en 2018 respecto de 2017 (Gráfico 23). 
Gráfico 21. Colombia: Número de inscripciones en Instituciones de

Educación Superior (IES) por sector, 2000 - 2018

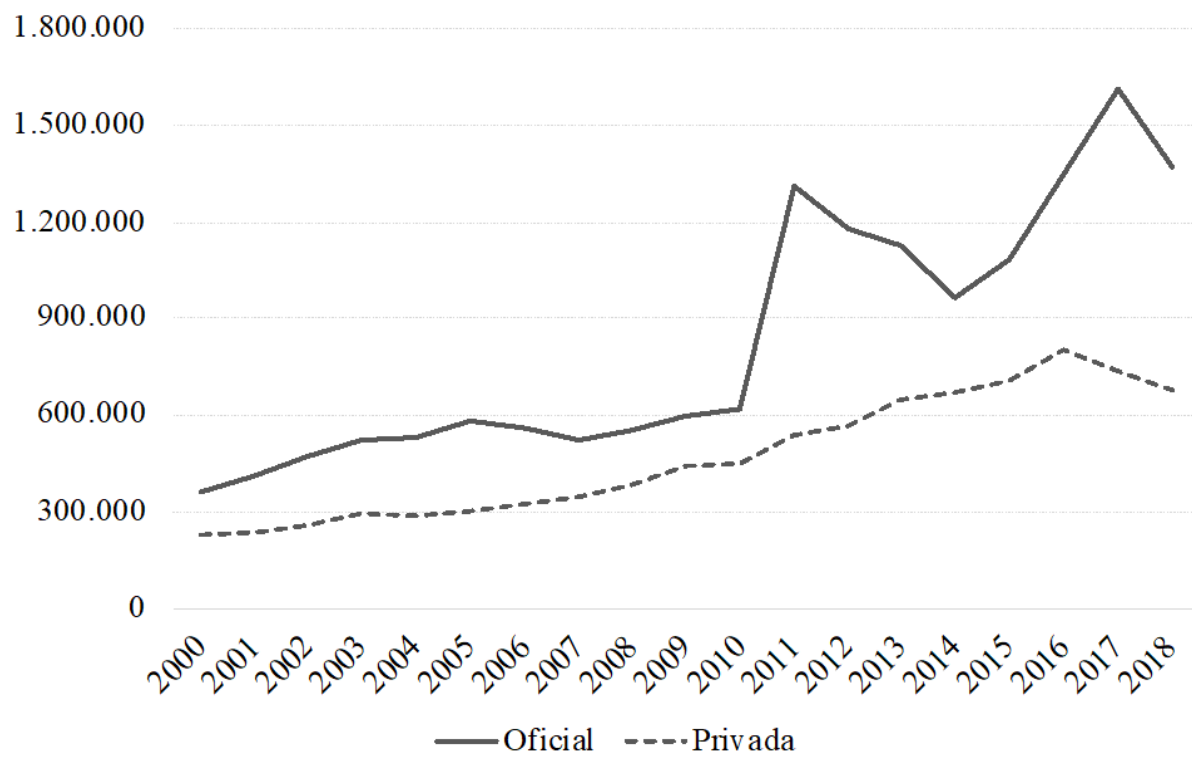

Fuente: Elaboración propia con base en datos del Sistema Nacional de Información de la Educación Superior (SNIES).

\section{Gráfico 22. Colombia: Número de inscripciones en} universidades por sector, 2000 - 2018

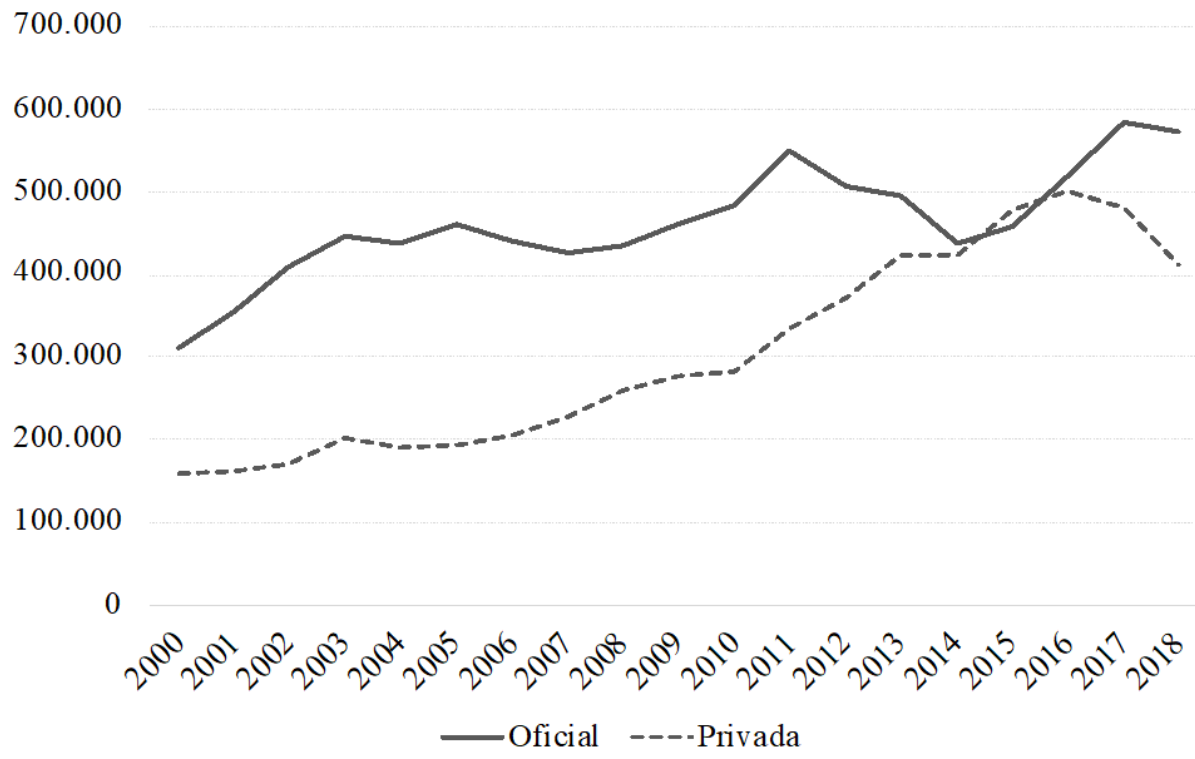

Fuente: Elaboración propia con base en datos del SNIES. 


\section{Gráfico 23. Colombia: Número de inscripciones en universidades por región, 2000 - 2018}

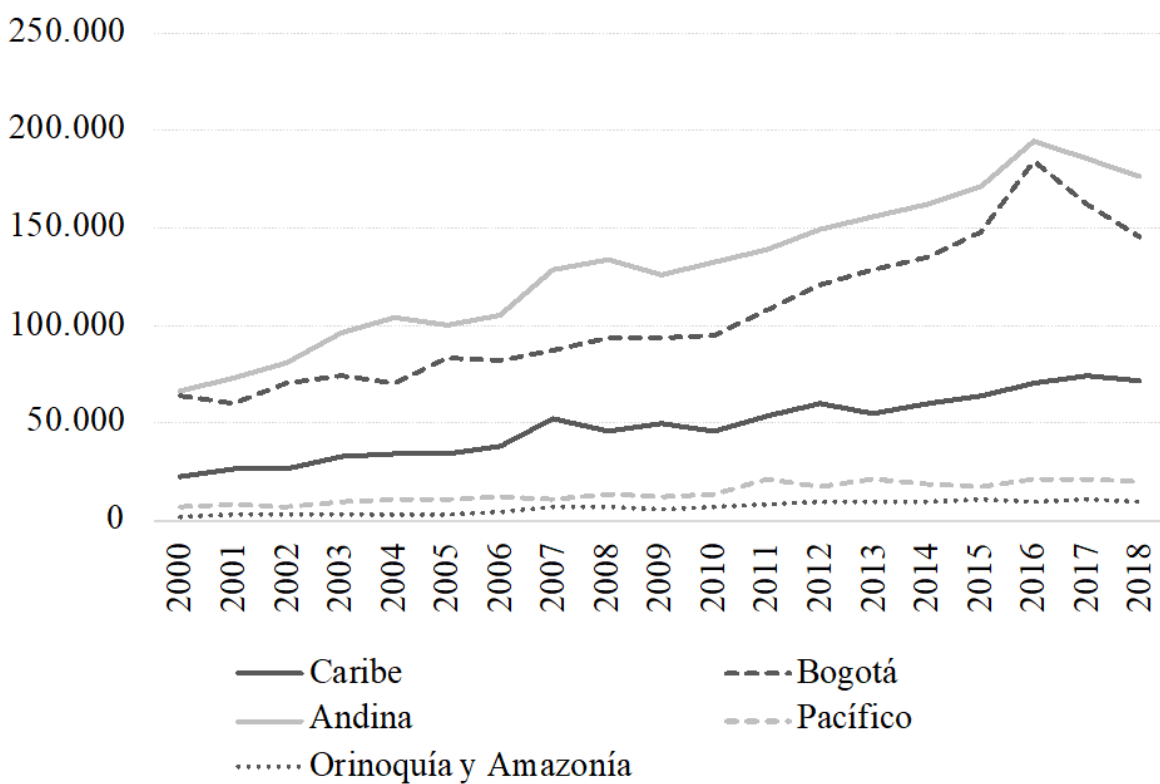

Nota: La región Caribe está conformada por los departamentos de Atlántico, Bolívar, Cesar, Córdoba, La Guajira, Magdalena, San Andrés y Sucre. La región Pacífica está conformada por los departamentos de Cauca, el Chocó y Nariño. La región Andina está conformada por los departamentos de Antioquia, Boyacá, Caldas, Cundinamarca, Huila, Norte de Santander, Quindío, Risaralda, Santander, Tolima y Valle del Cauca, y la ciudad de Bogotá. La Amazonía y la Orinoquía está conformada por los departamentos de Amazonas, Arauca, Caquetá, Casanare Guainía, Guaviare, Meta, Putumayo, Vaupés y Vichada.

Fuente: Elaboración propia con base en datos del SNIES.

En estos últimos dos años no solo disminuyó la demanda por educación superior, sino también el número total de estudiantes matriculados de nuevo ingreso en las universidades. El crecimiento de la matrícula de nuevos estudiantes en instituciones privadas fue de $-9 \%$ y de $-10 \%$ en 2017 y 2018, respectivamente. En instituciones públicas fue de $1 \%$ y -2 \% en los mismos años (Gráfico 24). En todas las regiones disminuyó el número de estudiantes matriculados en 2018, aunque nuevamente el efecto fue más moderado en la región Caribe, que decreció 2 \%, en contraste con Bogotá donde esta cifra fue 10 \% (Gráfico 25); y con Antioquia, que registró un crecimiento del 2 \% (Gráfico 26).

En los últimos cinco años, alrededor del $36 \%$ de los estudiantes de primer ingreso se matricularon en universidades en Bogotá. Más aun, entre 2017 y 2018, el 56 \% de la variación total de este tipo de estudiantes matriculados (29.659 estudiantes menos) se explica por la disminución en la capital del país (16.744 estudiantes menos), sobre todo en las universidades privadas (Gráfico 27). En ese sentido, aunque la caída de la matrícula en primer curso es un fenómeno generalizado a nivel nacional, el epicentro ha estado en Bogotá. 


\section{Gráfico 24. Colombia: Número de estudiantes matriculados en} primer curso universitario por sector, 2000 - 2018

350.000

300.000

250.000

200.000

150.000

100.000

50.000

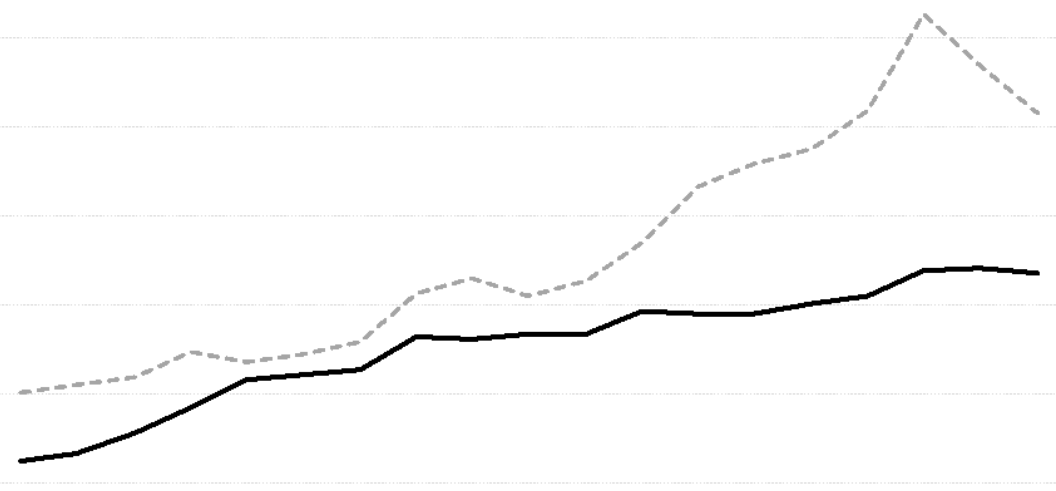

0

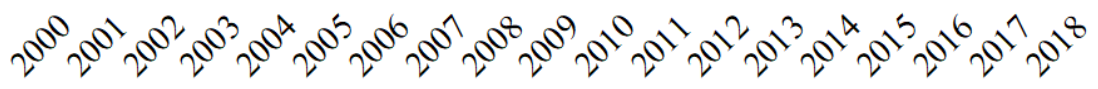

_-Oficial -----Privada

Fuente: Elaboración propia con datos del SNIES.

\section{Gráfico 25. Colombia: Número de estudiantes matriculados en} primer curso universitario por región, 2000 - 2018

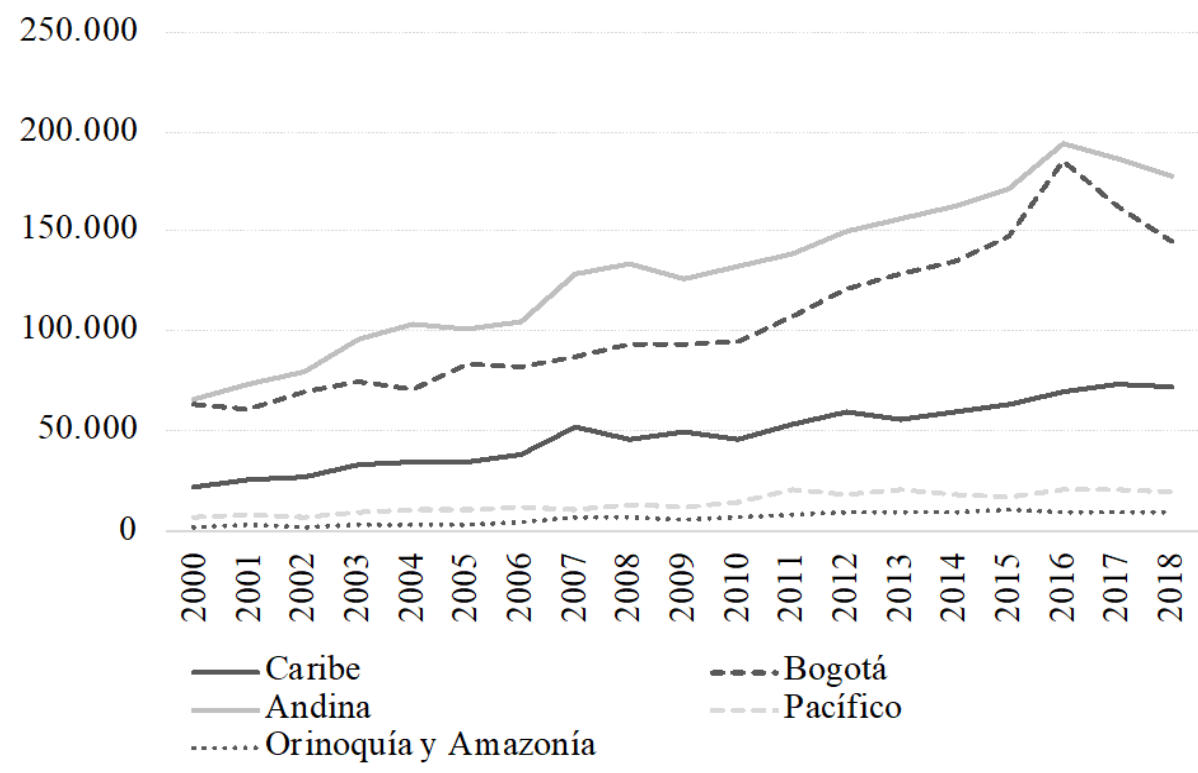

Fuente: Elaboración propia con datos del SNIES. 


\section{Gráfico 26. Colombia: Número de estudiantes matriculados en primer curso} universitario por principales departamentos, 2000 - 2018

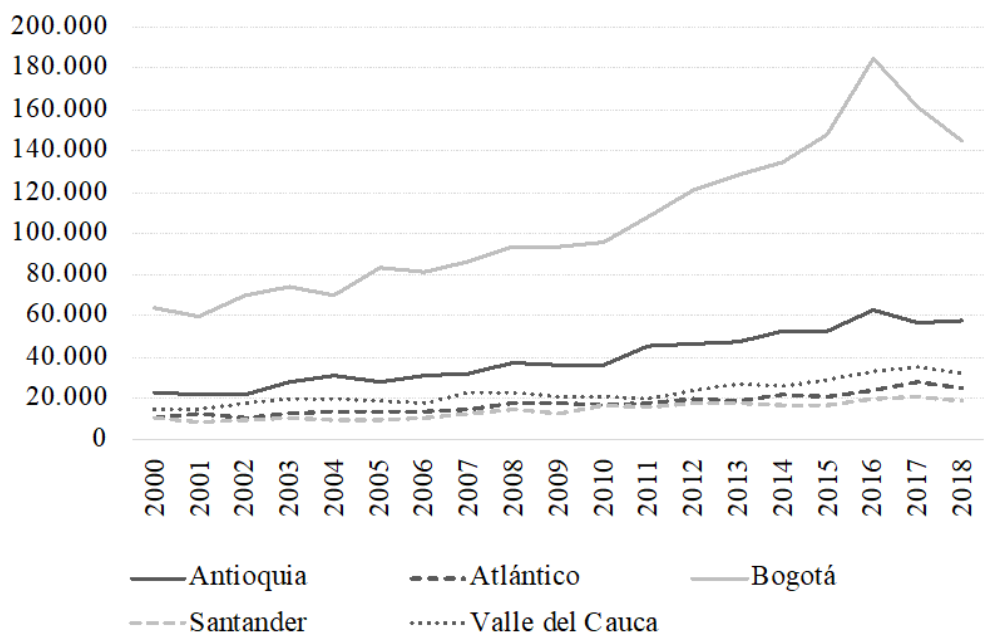

Fuente: Elaboración propia con datos del SNIES.

\section{Gráfico 27. Bogotá: Número de estudiantes matriculados en} primer curso universitario por sector, 2000 - 2018

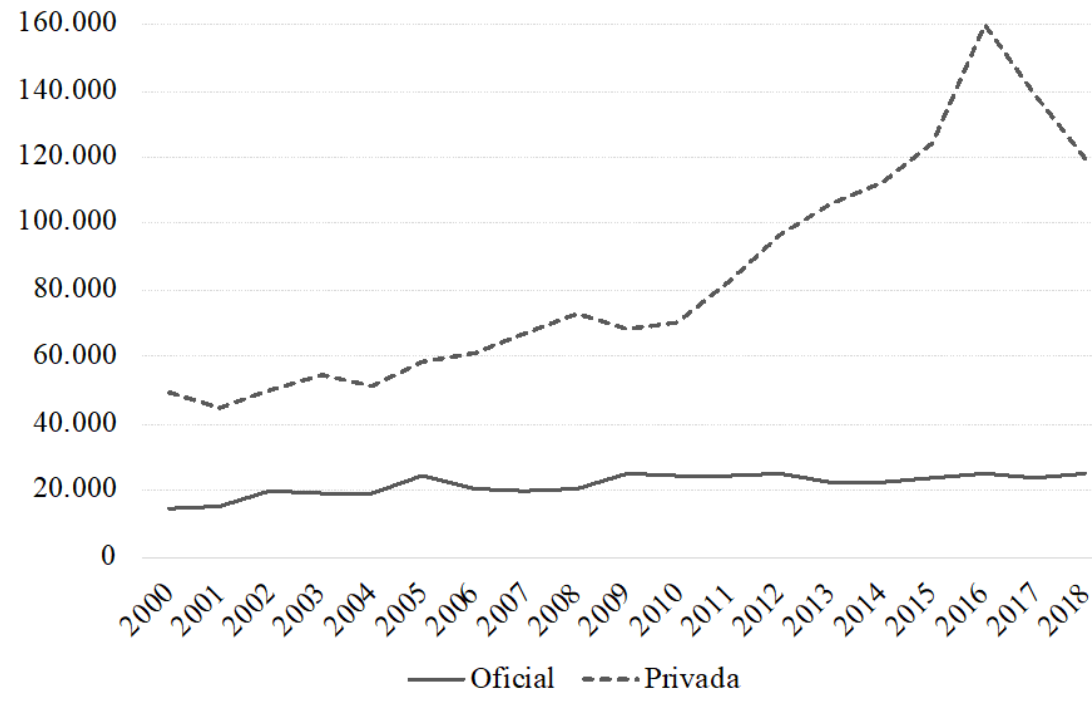

Fuente: Elaboración propia con datos del SNIES.

Las posibles explicaciones de este comportamiento reciente de las inscripciones y de la matrícula en las IES pueden ir desde causas coyunturales, como el costo de las matrículas, el bajo crecimiento económico y la creciente participación de la educación virtual, hasta causas más estructurales, como el decrecimiento de la demanda potencial debido a la dinámica demográfica. 
Es importante notar que hasta el momento las cifras mencionadas incluyen todas las modalidades de estudio universitario (a distancia tradicional, a distancia virtual y presencial), por lo que el comportamiento en la matrícula en una u otra modalidad ya está incorporado en las cifras agregadas. Esto descarta que la disminución que se observa desde 2017 se explique por una mayor demanda por educación virtual. No obstante, como se observa en el Gráfico 28, la educación virtual sí ha crecido en participación desde 2012. En 2011, significaba solo el 1 \% de la matrícula de primer ingreso en universidades, mientras que en 2018 representó el $10 \%$. Sin embargo, seguía siendo un mercado pequeño en comparación con la matrícula presencial. Contrario a las otras modalidades, el número de matriculados de primer ingreso en educación virtual en 2017 y en 2018 aumentó de 39.075 a 43.407. Las cifras no muestran que en los últimos tres años el crecimiento de la participación de la modalidad virtual haya sido en detrimento de la matrícula presencial, que de hecho ha crecido ligeramente en participación en años recientes. Más bien, ha tomado mercado de la matrícula a distancia tradicional, que cayó durante esos años.

Otra de las hipótesis coyunturales se relaciona con el incremento en los costos de matrícula. Aunque probar esta hipótesis para Colombia se puede abordar en próximas investigaciones, existe evidencia en la literatura internacional que soporta que los costos de matrícula son un determinante de la elección de la institución mas no de la disposición a demandar educación superior (Sojkin, et al., 2011). Otros estudios argumentan que el efecto de los costos en la elección de universidades es pequeño y priman otros

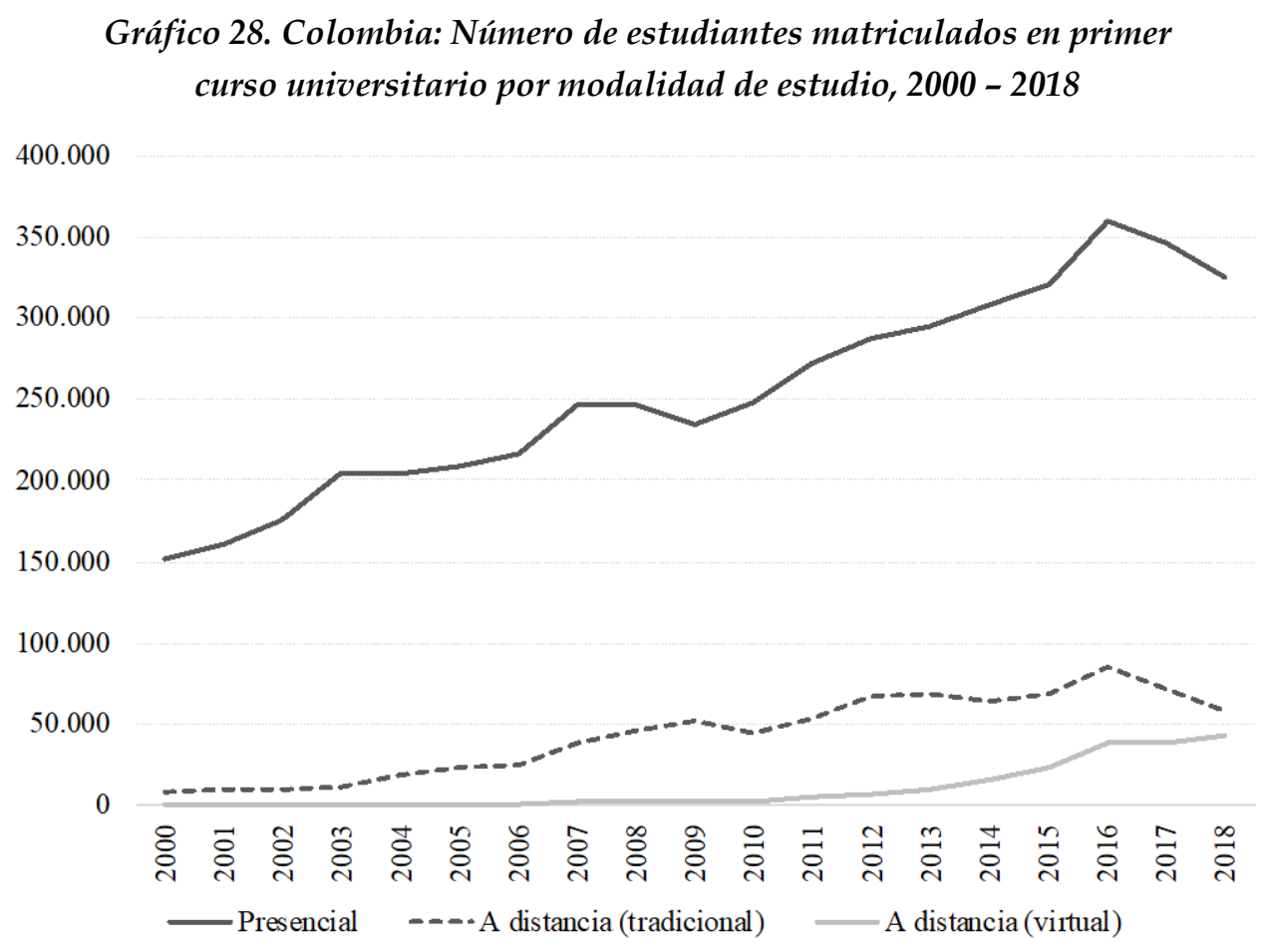

Fuente: Elaboración propia con datos del SNIES. 
determinantes como el apoyo de la familia, la imagen de la universidad y la oferta de programas (Rudhumbu, et al., 2017). Çokgezen (2014) encuentra que el impacto del costo de matrícula es mayor para los estudiantes de universidades públicas, mientras que para los estudiantes de universidades privadas es más importante la reputación de la institución. Este argumento va en línea con aquel propuesto por McDuff (2007), quien argumenta que los estudiantes están dispuestos a pagar altos costos de matrícula a cambio de una educación de alta calidad. Para el caso colombiano, existen pocos estudios entorno a la importancia de los costos en la demanda por educación superior. Además, los bachilleres provenientes de estratos bajos y medios tienden a optar por universidades públicas, mientras que los bachilleres de estratos altos seleccionan universidades privadas (Ramírez, 2008).

En lo que respecta a la hipótesis del desempleo, la teoría del capital humano argumenta que la elección de estudiar en lugar de trabajar responde a una estimación del valor presente de la relación costo/beneficio. En este sentido, si el desempleo aumenta, como en efecto ha sucedido en Colombia desde 2016 hasta 2018 (de $10 \%$ a 10,8 \%), disminuyen los costos de oportunidad de estudiar y, por tanto, más estudiantes deberían demandar educación universitaria.

En general, hay poca evidencia en torno a las hipótesis coyunturales, y los datos, así como el análisis histórico del último siglo en Colombia, sugieren que la causa del decrecimiento de la demanda en las universidades es de tipo estructural y está ligada al cambio demográfico. El Gráfico 16 muestra que las cohortes por año de nacimiento de estudiantes que presentan la prueba Saber 11 crecieron de forma sostenida hasta 1996. Paralelamente, desde 2017, se muestra un decrecimiento de las inscripciones y las matrículas en universidades y otras IES. Aunque en otros momentos del tiempo ha caído la matrícula, esta última disminución coincide también con cohortes más pequeñas de graduados de bachillerato. Que los estudiantes que se presentan a las IES tengan entre 14 y 21 años significa que quienes demandan un cupo en 2017 nacieron entre 1996 y 2003. Según las pruebas Saber 11 estos son los años en los que comienza a disminuir el número de estudiantes que ingresa y posteriormente termina el colegio. Esto refuerza la hipótesis estructural, y aunque es un fenómeno reciente, la evolución de la transición demográfica en Colombia y la demanda universitaria de los últimos años son evidencia de que las universidades se enfrentarán en los próximos años a los retos que supone el creciente envejecimiento de la población.

No quiere decir esto que el bajo crecimiento económico, la educación virtual, los programas de gobierno y el costo de la matrícula no tengan efectos sobre el número de estudiantes que ingresan a las universidades. Son importantes para explicar, ante determinadas coyunturas, las variaciones de corto y mediano plazo de la demanda por educación superior. Más bien, sugiere que, independiente de cómo se comporten estas variables, existe un cambio estructural en la población colombiana en la que la población en edad escolar es cada vez menor, y esto afecta la matrícula en las instituciones educativas. No obstante, estos determinantes próximos de la demanda educativa (ingreso esperado, desempleo, modalidad de la 
educación, subsidios) son clave para que las universidades y el Gobierno reduzcan el impacto negativo de la transición demográfica.

\section{RETOS EN LA EDUCACIÓN SUPERIOR POR CAUSA DE LA TRANSICIÓN DEMOGRÁFICA}

Aunque la base de la pirámide poblacional en Colombia y en el mundo es cada vez más reducida, la brecha entre la demanda potencial y la demanda efectiva en el país aún es significativa. Cerca de la mitad de los jóvenes de entre 17 y 21 años no accede a la educación superior y, en consecuencia, no es posible declarar escasez absoluta de jóvenes para ingresar a las universidades. De hecho, el principal problema no es la población que se inscribe a la universidad, y tampoco el cambio en las motivaciones de los estudiantes, sino el tamaño de la población que queda por fuera del sistema. Por tanto, uno de los principales retos es la ampliación de la cobertura en este nivel.

En este sentido, a pesar de que la reducción del número de estudiantes en las universidades es en parte un resultado de la transición demográfica, las políticas efectivas que aumenten la cobertura pueden compensar durante algunas décadas este fenómeno, tal como sucedió entre 1950 y 1990 con la cobertura en la educación básica y media. En ese nivel, la matrícula siguió creciendo, aunque las cohortes de jóvenes ya habían empezado a decrecer. Además, la disminución de la población en edad escolar disminuye la demanda de cupos sobre el sistema educativo y, en consecuencia, se liberan recursos del sector público que se pueden utilizar para aumentar la cobertura y la calidad en los niveles de secundaria, media y superior.

Grawe (2018) estudió la demografía y la demanda por educación superior en los Estados Unidos, y proyectó una década de crecimiento modesto en la asistencia a las universidades seguida de una gran contracción entre 2025 y 2029. Lo primero sucede mientras la generación que representa "la escasez de nacimientos" alcanza la edad para entrar en las universidades. Este período transitorio en Colombia, que tiene una tasa de fecundidad más baja que los Estados Unidos, puede haber llegado a su fin. Las generaciones que representan la escasez de nacimientos con mejores oportunidades de ingresar y terminar el colegio (después de 1997) ya han alcanzado la edad para entrar en la universidad. Pero, como se mencionó antes, los retos en cobertura en la educación superior nos colocan en un escenario distinto al estadounidense.

La cobertura debe ampliarse, sobre todo en la población de ingresos más bajos, entre aquellos jóvenes sin recursos suficientes para pagar las matrículas o financiar su sostenimiento. A menudo esta población tiene desventajas para acceder a las becas de alto rendimiento debido a la baja calidad de la educación básica y media que han recibido. Algunas de las recomendaciones en esta línea implican fortalecer el crédito educativo, ampliar los programas públicos e incrementar la participación del sector privado y la gestión de las universidades en la consecución de mayores recursos para becas estudiantiles. 
En lo que respecta a las instituciones universitarias, e instituciones educativas en general, el cambio estructural de la población lleva a nuevos retos en calidad y metodologías de la educación. Por un lado, hay que ampliar y transformar los servicios educativos que ofrecen de forma tal que respondan a los intereses de los estudiantes y a los requerimientos en el mercado laboral, es decir, trabajar en la pertinencia de la educación. Por otro lado, en la medida en que se reduce la demanda, aumenta la competencia entre universidades para atraer estudiantes. La evidencia a nivel mundial sugiere que el impacto de los cambios demográficos es mucho menor o nulo en las instituciones de alta calidad. Grawe (2018) encuentra efectos diferenciales según la ubicación geográfica de las universidades y su posición en el ranking. En particular, sostiene que en los Estados Unidos la demanda por las instituciones élites (en el top 50) aumentará 14 \% entre 2012 y 2029. Grawe argumenta que estas instituciones son nichos de mercado pequeños con bajas tasas de admisión. En contraste, las instituciones que basan su matrícula en la atracción de los estudiantes cercanos a su localización sentirán en mayor medida los efectos de la reducción de los nacimientos.

\section{CONCLUSIONES}

La transición demográfica ha afectado en los años más recientes el tamaño de las cohortes que se gradúan de bachillerato. En Colombia se está presentado una caída de la tasa de natalidad que está llevando a que el número de estudiantes que culminan grado 11 sea cada vez menor. Como es muy probable que los estudiantes de estratos socioeconómicos más altos sean los que presentan mayor caída en el tamaño de las cohortes, este proceso seguirá por unos años más. El hecho de que son los estudiantes de estratos socioeconómicos más altos los que muestran mayor caída en el tamaño de las cohortes se confirma por el hecho de que la reducción en los inscritos es mayor en las universidades privadas que en las públicas.

Por otro lado, hay un claro patrón centro-periferia en la caída del tamaño de las cohortes que se está sintiendo en las universidades colombianas. La caída se ha observado, sobre todo, en Bogotá y la región Andina, y muy poco o nada en la periferia del país, la región Caribe y el litoral Pacífico. Esto se explica porque la periferia está rezagada económicamente con respecto al centro del país y, por tanto, los factores estructurales que afectan la caída de la natalidad, como el grado de educación y la participación de las mujeres en el mercado laboral, se encuentra todavía por debajo de los promedios nacionales.

Ante el panorama actual, hay que resaltar que en Colombia la cobertura en educación superior, del 52,8 \% en 2018, es aún muy baja. Por tanto, la caída en la demanda actual representa una oportunidad para aprovechar la infraestructura ya existente para ampliar la cobertura. En este sentido, hay que considerar que en Colombia alrededor del $50 \%$ de los estudiantes universitarios asisten a instituciones privadas. Muchos estudiantes no acceden a estas universidades por no contar con los recursos necesarios. Por otro lado, las universidades públicas no tienen los cupos disponibles ni los recursos para poder ampliarse 
rápidamente. Por ende, el reto es cómo financiar con créditos o becas parciales a esos estudiantes potenciales que no tienen los recursos para pagar sus estudios universitarios. Esos créditos podrían ser parcialmente condonables si se dan con recursos del Estado o créditos con intereses más bajos si son con recursos del sector privado. Además, al terminar de estudiar, el pago debe ser contingente con el ingreso. Por ejemplo, suspender el cobro si el profesional está desempleado. Este tipo de políticas promueven la igualdad de oportunidades en la educación.

\section{REFERENCIAS}

Barón R., Juan David (2003), “¿Qué sucedió con las disparidades económicas regionales en Colombia entre 1980 y el 2000?", Documentos de Trabajo sobre Economía Regional, No. 38.

Bonet Morón, Jaime, y Adolfo Meisel Roca (1999), “La convergencia regional en Colombia: Una visión de largo plazo, 1926 - 1995". En Adolfo Meisel Roca (editor), Regiones, ciudades y crecimiento económico en Colombia, Bogotá: Banco de la República.

Centro Latinoamericano de Demografía (CELADE) (1996), Impacto de las tendencias demográficas sobre los sectores sociales en América Latina. Contribución al diseño de políticas y programas, Santiago de Chile: CELADE.

Chackiel, Juan (2004), “La dinámica demográfica en América Latina”, Población y Desarrollo, No. 52.

Clark, Gregory (2007), A Farewell to Alms: A Brief Economic History of the World, Princeton: Princeton University Press.

Çokgezen, Murat (2014), "Determinants of University Choice: A Study on Economics Departments in Turkey", Yüksekögretim Dergisi, Vol. 4, No. 1. DOI: https:// doi.org/10.2399/yod.14.002.

Comisión Económica para América Latina y el Caribe (CEPAL) (2008), Transformaciones demográficas y su influencia en el desarrollo en América Latina y el Caribe, Santo Domingo: CEPAL.

El Tiempo (2019), “¿Por qué cayeron las matrículas en universidades?”, septiembre 30.

Flórez, Carmen Elisa, y Olga Lucía Romero (2010), "La demografía de Colombia en el siglo XIX". En Adolfo Meisel Roca y María Teresa Ramírez (editores), Economía colombiana del siglo XIX, Bogotá: Fondo de Cultura Económica.

Flórez N., Carmen Eliza (2000), Las transformaciones sociodemográficas en Colombia durante el siglo XX, Bogotá: Banco de la República de Colombia.

Galvis, Luis Armando, y Adolfo Meisel (2012), “Convergencia y trampas espaciales de pobreza en Colombia: Evidencia reciente", Documentos de Trabajo sobre Economía Regional, No. 177.

Grawe, Nathan D. (2018), Demographics and the Demand for Higher Education, Baltimore: Johns Hopkins University Press.

Livi-Bacci, Massimo (2017), A Concise History of World Population, Malden: John Wiley \& Sons.

Martínez Gómez, Ciro (2013), Descenso de la fecundidad, bono demográfico y crecimiento económico en Colombia 1990-2010, Bogotá: Ministerio de Salud.

McDuff, DeForest (2007), "Quality, Tuition, and Applications to In-State Public Colleges", Economics of Education Review, Vol. 26, No. 4. DOI: https:/ / doi.org/10.1016/j.econedurev.2006.04.003

Meisel R., Adolfo, y Angela Granger S. (2019), “¿Atrapados en la periferia? Brechas de calidad en la educación en Colombia: Pruebas Saber 11 (2000-2018)", Documentos, No. 36.

Meisel Roca, Adolfo, y María Teresa Ramírez Giraldo (editores) (2010), Economía colombiana del siglo XIX, Bogotá: Fondo de Cultura Económica.

Mejía, Daniel, María Teresa Ramírez, y Jorge Tamayo (2009), “Transición demográfica en Colombia”, Reportes del Emisor, No. 116.

Portafolio (2018), “El alto costo de las matrículas universitarias en Colombia”, febrero 25. 
Ramírez G., María Teresa, y Juana Patricia Téllez C. (2006), “La educación primaria y secundaria en Colombia en el siglo XX", Borradores de Economía, No. 379. DOI: https:/ / doi.org/10.32468/ be.379

Ramírez Hassan, Andrés (2008), “Modelos de elección discreta. Una aplicación a la demanda por cupos universitarios en ciudades intermedias de Colombia", Revista Universidad EAFIT, Vol. 44, No. 152.

Rudhumbu, Norman, Avinash Tirumalai and Babli Kumari (2017), “Factors that Influence Undergraduate Students' Choice of a University: A Case of Botho University in Botswana”, International Journal of Learning and Development, Vol. 7, No. 2. DOI: https://doi.org/10.5296/ijld.v7i2.10577.

Searchinger, Tim, Craig Hanson, Richard Waite, Sarah Harper, George Leeson, and Brian Lipinski (2013), “Achieving Replacement Level Fertility", Working Paper, Installment 3 of Creating a Sustainable Food Future.

Semana (2020), “¿Por qué caen las matrículas en las universidades en Colombia?”, enero 16.

Sojkin, Bogdan, Paweł Bartkowiak, and Agnieszka Skuza (2011), “Determinants of Higher Education Choices and Student Satisfaction: The Case of Poland", Higher Education, No. 63. DOI: https:/ / doi.org/10.1007/s10734-011-9459-2.

Todaro, Michael, and Stephen Smith (2012), Economic Development, 11th Edition, UK: Pearson Addison Wesley. 


\section{ANEXOS}

Anexo 1. Colombia: Índice de dependencia, 1960 - 2018

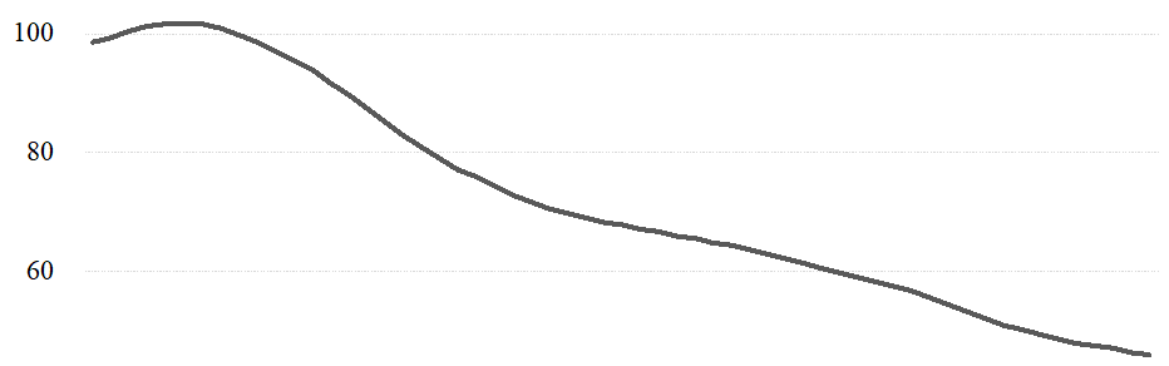

40

20

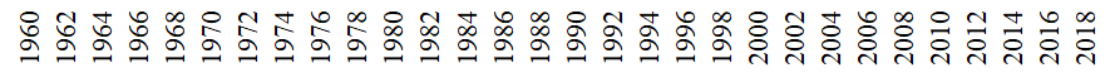

Fuente: Elaboración propia con base en datos del Banco Mundial.

Anexo 2. Estudiantes entre 14 y 18 años que presentaron las pruebas Saber 11 entre 2000 y 2018 por año de nacimiento y región

(crecimiento indexado: $1984=100$ )

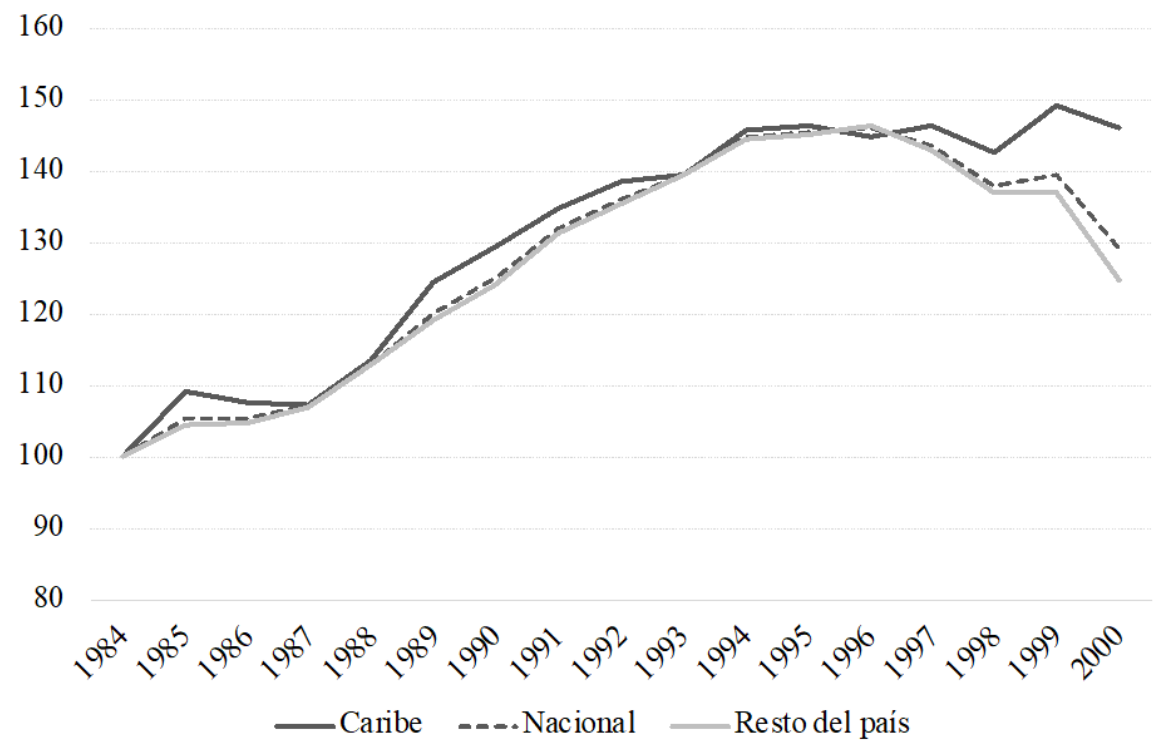

Fuente: Elaboración propia con base en datos del Icfes. 


\section{Anexo 3. Colombia: Número de inscripciones en IES por nivel de formación}

(crecimiento indexado: $2000=100)$

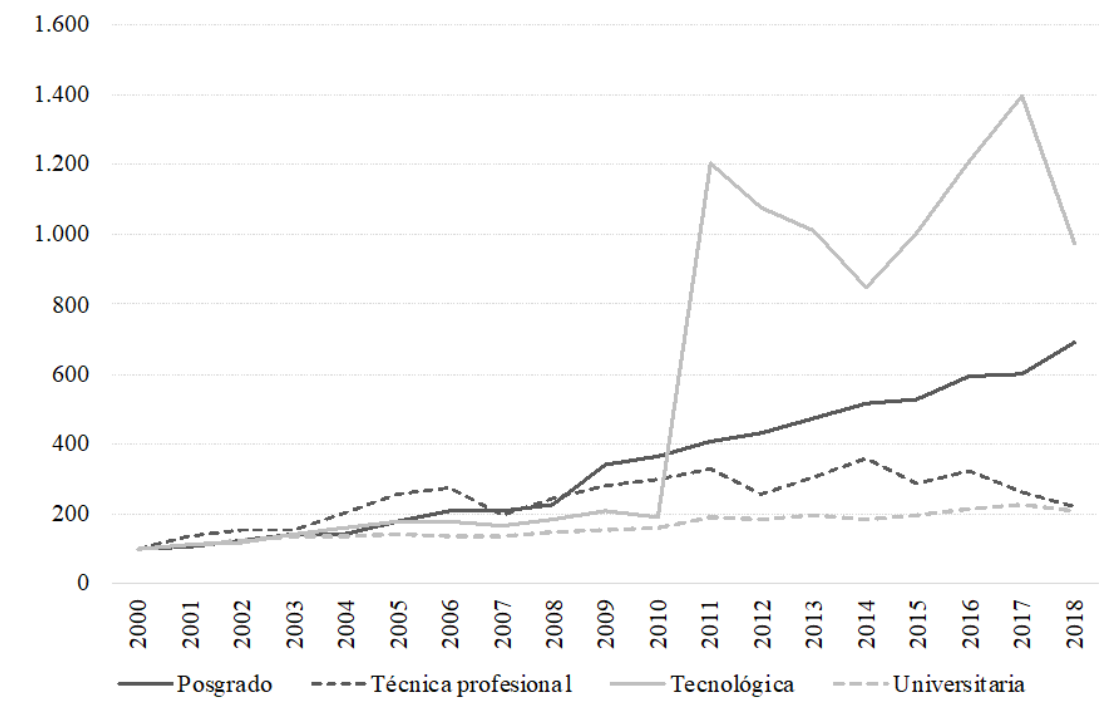

Nota: EL crecimiento en las inscripciones y matricula a educación tecnológica en 2010 está relacionado con un incremento de más del 30\% en el presupuesto del Servicio Nacional de Aprendizaje (SENA) durante este año.

Fuente: Elaboración propia con base en datos del SNIES.

\section{Anexo 4. Colombia: Número de estudiantes matriculados en primer curso de educación superior por nivel de formación \\ (crecimiento indexado: $2000=\mathbf{1 0 0}$ )}

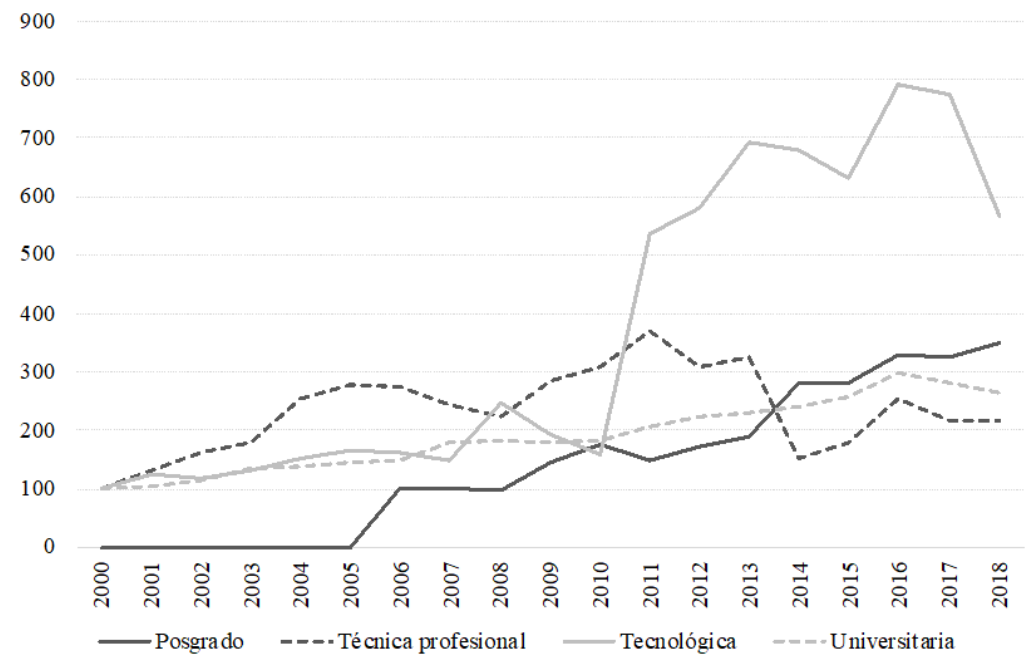

Nota: Entre 2000 y 2005 no existe información de matriculados en posgrados (excepto para 2001).

Fuente: Elaboración propia con base en datos del SNIES.

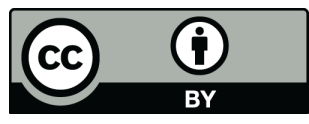

(C) 2020 by the authors. Licensee Economía \& Región, Cartagena, Colombia. This article is an open access article distributed under the terms and conditions of the Creative Commons Attribution (CC BY) license (http:/ / creativecommons.org/licenses/by/4.0/). 